\title{
On the precision of X-ray source parameters estimated from ROSAT data ${ }^{\star}$
}

\author{
F. G. Boese
}

\author{
Max-Planck-Institut für extraterrestrische Physik, 85741 Garching, Germany \\ e-mail: gub@mpe.mpg.de
}

Received 10 July 2003 / Accepted 14 May 2004

\begin{abstract}
The precision of the point source parameters, i.e. those for source position, source counts and source extension obtained via Maximum Likelihood Estimation from ROSAT data, is investigated. The various categories of error in X-ray observatories are identified. An analytic perturbation analysis is set forth. This means in the ROSAT case a perturbation analysis of the likelihood function with respect to the perturbed quantity being scrutinized. The background distribution and point spread function of the telescope-detector unit belong to these quantities. ROSAT observations are simulated and subjected to data analysis with the ROSAT software. The parameter space spanned by the point source parameters is explored in this way. Common summarizing statistics, namely mean value and standard error for the empirical distributions of the source parameters obtained in simulation runs, are taken as estimates for the unknown true precision counterparts. The precision for the errors of the source parameters as determined in the ROSAT data analysis are treated in the same way. In this sense, the source count imprecision is below 2 counts (for ROSAT typical sources and background), the directional positional precision below $2^{\prime \prime}$, and the source extent imprecision below 3" for the ROSAT PSPC detector. For the HRI detector, the positional precision figures are better. An alternative approach to the precision issue by cross correlating the ROSAT All-Sky Bright Source Catalogue with the optical TYCHO star catalogue is taken in order to assess the over-all precision. Inference on the positional accuracy for the ROSAT sources along with the quality of the accompanying ROSAT position errors is made. The positional errors determined in this way are about $8^{\prime \prime}$ larger than the simulation counterpart. This is in qualitative agreement with the $6^{\prime \prime}$ surplus added for the random attitude errors and all other known or less well known error sources. In this sense, the catalogue positional errors are compatible with the positional precision found in the simulations.
\end{abstract}

Key words. X-ray: general - space vehicles: instruments - techniques: image processing

\section{Introduction}

This paper is a further step to the completion of the documentation of the technical fundamentals of the processing of ROSAT data. This article is devoted to precision issues. Reliability questions of the kind: to what degree can one trust the numbers in the source lists issued for celestial direction, photon count rate, and extension of a specific ROSAT source are of prime interest for source identifications in catalogues from different wavelength regimes and source comparisons with respect to time, celestial direction, and source strength at observations at the same wavelength.

Such problems will be addressed in two forms, in an instrument-independent form and, as the title promises, in the context of ROSAT data and ROSAT data processing.

In Boese (2000), henceforth Paper I, the point spread functions (PSF) in effect in the ROSAT data processing were described. In a subsequent paper, Boese \& Döbereiner (2001),

^ Dedicated to H.-U. Zimmermann on the occasion of his retirement.
Paper II, the estimation method used for the ROSAT data, Maximum Likelihood Estimation (MLE), was documented. The precision topics were touched upon only in a subsection of the latter paper. It was felt that the precision problem deserves a treatment of its own.

The ROSAT satellite accomplished the most successful X-ray observing mission up until now (in terms of new sources).

The ROSAT satellite was launched on June 1, 1990 into a circular orbit of altitude $580 \mathrm{~km}$, with an inclination of $53^{\circ}$, an orbit period of $96 \mathrm{~min}$, and a precession period of 66 days. The last observation was made on December 18, 1998. The ROSAT detectors were (a) the Positional Sensitive Proportional Counter (PSPC); (b) the High Resolution Imager (HRI); and (c) the Wide Field Camera (WFC). Two telescopes were in operation: the larger one for the PSPC and HRI, the smaller for the WFC. The ROSAT detectors PSPC and HRI register single X-ray photons. The physical quantities of individual Xray photons recorded are (a) the time of photon arrival; (b) the impact location in the detector; (c) the amplitude of the 


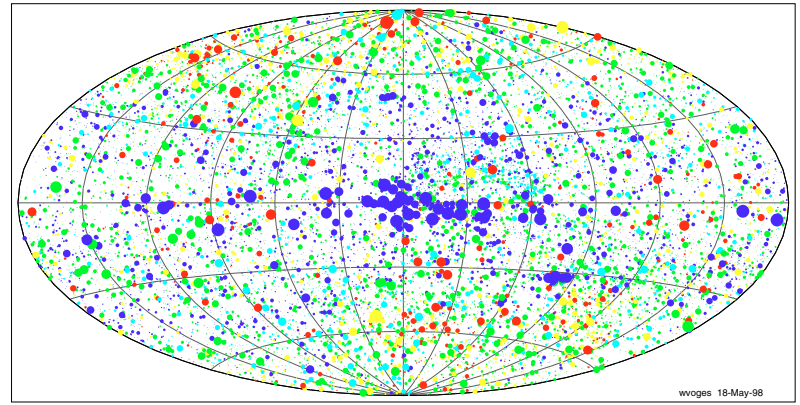

Fig. 1. The 18811 ROSAT All-Sky Survey Bright Sources in the energy band $[0.1,2.4] \mathrm{keV}$ with a Galactic II coordinate net. The source radii are $\propto \log _{10}$ (source counts), grey scale $\propto$ (source temperature).

detector signal, and (d) the infalling direction ${ }^{1}$, see Trümper (1983). Depending on the ROSAT observation mode, all-sky survey or pointed observation, the instrument's optical axis is, relative to the observed target, nominally fixed or moving in time. During an observation time interval, a number of photons is collected. After data preparation, the observed sample forms the data set to be analyzed.

The majority of all observed celestial sources are point sources. The capacity of analyzing point sources with respect to (a) X-ray flux; (b) celestial direction and (c) extent (if larger than but akin to point sources) allows one thus to treat a large percentage of all observed sources routinely.

The ROSAT data analysis systems are (a) the Standard Analysis Software System (SASS), cf. Voges et al. (1992), and (b) the Extended Scientific Analysis System (EXSAS), see Zimmermann et al. (1998, 1992). The basic data processing is performed with SASS. The EXSAS software system assists in analyzing ROSAT observational data scientifically. It is distributed to more than 300 observers in 75 institutes worldwide. The latest EXSAS version released is of October 2003.

Up to the end of 2002, more than 5400 scientific papers based on ROSAT data appeared in journals or conference reports. The ROSAT publication rate from February 1999 to December 2001 amounts to 27 per month in refereed journals and 44 including non-refereed articles.

Among the many ROSAT catalogues are the ROSAT AllSky Survey Bright Source Catalogue with 18811 sources, see Fig. 1, and the Source Catalogue from Pointed PSPC Observations with 74301 sources, see Voges et al. (1999). A considerable percentage of all related data analysis was carried out with the SASS or EXSAS analysis systems - an imperative reason to report thoroughly on the achieved precision.

In Sect. 2, the various error sources will be compiled. The effect of these errors on the source estimation is analytically investigated in Sect. 3. Source simulation as a means to explore the source parameter space is the topic of Sect. 4. Point sources as determined by ROSAT software from ROSAT data and as found in a standard catalogue will be compared statistically in Sect. 5. Source detection examples will be provided in Sect. 6. Section 7 contains the discussion of the results. Conclusions are given in Sect. 8 .

\footnotetext{
1 This direction is not recorded per photon but evaluated per photon. The attitude of the optical axis is thereby used.
}

Table 1. The ROSAT observation modes (OMode), the number of registered X-ray photons (\#Photons), the related observation times (Time) in seconds, and the number of observations (\#Obs) per mode. PSPC-S = ROSAT all-sky survey with PSPC, PSPC-P = ROSAT pointed observations with PSPC, HRI = ROSAT pointed observations with HRI.

\begin{tabular}{lrrr}
\hline \hline OMode & \#Photons & Time [s] & \#Obs \\
\hline PSPC-S & 102181114 & 642732 & - \\
PSPC-P & 523612984 & 32404595 & 4991 \\
HRI & 97650517 & 59917454 & 4721 \\
\hline Total & 723444615 & 92964781 & 9713 \\
\hline
\end{tabular}

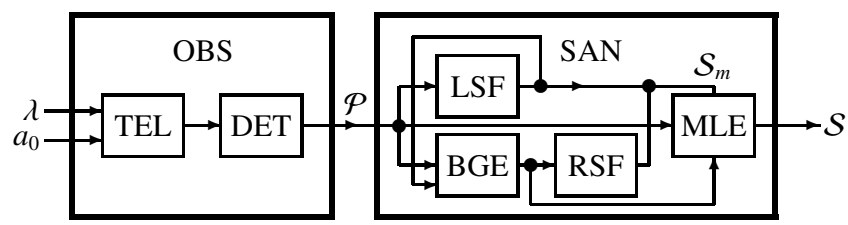

Fig. 2. The ROSAT source finder. OBS = observatory, SAN = source analyzer, TEL $=\mathrm{X}$-ray telescope, $\mathrm{DET}=$ detector, $\mathrm{LSF}=$ local source finder, $\mathrm{BGE}=$ background estimator, $\mathrm{RSF}=$ refined source finder, MLE $=$ maximum likelihood estimator, $\lambda=$ celestial photon density, $a_{0}=$ nominal satellite attitude, $\mathcal{P}=$ photon sample, $\mathcal{S}_{\mathrm{m}}=$ merged preliminary source list, $\mathcal{S}=$ source list.

During its $8 \frac{1}{2}$ years life time span, ROSAT has harvested from the Cosmos about 723 million X-ray photons, stemming from (1) the All-Sky Survey with the PSPC; (2) the 4991 pointed observations with the PSPC, and (3) from the 4721 pointed observations with the HRI in the related observation periods. See Table 1 for the contributions per each ROSAT observation mode and in total.

\section{The error sources}

In this section we trace the error sources limiting the precision of the final estimates of the single source parameters and also determining the size of the associated error bars.

The system under consideration consists of the X-ray photons acquiring observatory OBS in hardware followed by the source analyzer SAN in software in Fig. 2. The arrows indicate the photon flow or the data flow directions. The imaging $\mathrm{X}$-ray observatory is composed of one or more Wolter type I telescopes and one of several detectors in the focal plane. The detector performs single photon measurements and delivers photon data. A further output (not shown in Fig. 2) of the observatory OBS and input to the source analyzer SAN is the measured attitude time series $a(t)$.

The system input consists of the nominal attitude vector $a_{0}(t), t \in[\underline{T}, \bar{T}]=: \boldsymbol{T}$, and the celestial photon density $\lambda$ as a function of time, celestial direction and energy. During the observation interval $\boldsymbol{T}$, the observatory samples from $\lambda$ within the telescope's field of view ${ }^{2}$ around the pointing direction $p_{0}(t)$. Upon completion of the observation, a photon sample $\mathcal{P}$ is at

\footnotetext{
2 The largest disks within the field of view have the radii $57^{\prime}$ for PSPC and 20' for HRI.
} 
disposal on Earth. The off-line source analyzer SAN comprises in EXSAS the local source finder LSF, the background estimator BGE, the refined source finder RSF, and the final maximum likelihood source estimator MLE. The union of the source lists from LSF and RSF is the merged list $\mathcal{S}_{\mathrm{m}}$. All boxes of SAN are described in Paper II. The analyzer outputs a source list $\mathcal{S}$ with 34 columns containing for each source found the 4-vector $p$ of the source parameters; namely the number of source photons $\Lambda_{\mathrm{S}}$, the source direction $\left(X_{\mathrm{S}}, Y_{\mathrm{S}}\right)$ and the source extent parameter $v_{\mathrm{S}}$ along with the 4-vector $\sigma$ of the associated standard errors and the $\log$-likelihood $L_{\mathrm{S}}$ of source existence ${ }^{3}$,

$$
\begin{aligned}
\mathcal{S}^{\prime} & :=\left(L_{\mathrm{S}}, p, \sigma\right), \\
p & :=\left(\Lambda_{\mathrm{S}}, X_{\mathrm{S}}, Y_{\mathrm{S}}, \nu_{\mathrm{S}}\right), \quad \sigma:=\left(\sigma_{\Lambda_{\mathrm{S}}}, \sigma_{X_{\mathrm{S}}}, \sigma_{Y_{\mathrm{S}}}, \sigma_{v_{\mathrm{S}}}\right) .
\end{aligned}
$$

The 25 further ancillary quantities in the remainder source list $\mathcal{S} \backslash \mathcal{S}^{\prime}$ are not so important for our purposes.

In each of the boxes in Fig. 2 arise errors of different nature which we group into the four categories,

$$
\begin{aligned}
& \text { 1. technological errors; } \\
& \text { 2. modelling errors; } \\
& \text { 3. methodological errors; } \\
& \text { 4. statistical errors. }
\end{aligned}
$$

Uncertainties, deviations, realization losses, design limitations and the like, collectively named as errors, are unavoidable. Consequently, in terms of fully specified systems from Fig. 2, we have to take into consideration a whole family of such systems. This family is formed around a nominal system determined by a nominal design vector $q_{0}$ ranging in a design space $\boldsymbol{Q}$. When these quantities undergo variations $\delta q$, the said family is formed. In the case of small variations $\delta q$ the space $\boldsymbol{Q}$ is only a small neighbourhood around the nominal design vector $q_{0}$. It is usually less difficult to treat small variations than those of arbitrary size. We list varying quantities in the next four subsections.

\subsection{Technological errors}

Inaccuracies introduced by technological limitations at various places in the observatory propagate through the system and are, eventually, found in the source list $\mathcal{S}$. We note the error contributions due to (a) imprecise satellite attitude; (b) finite detector resolution; (c) imprecise time measurement.

We select an appropriate celestial coordinate system on the unit sphere $\boldsymbol{S}^{2}$ and a time frame consisting of an origin and a time unit. The former is the equatorial for epoch 2000 in the case of ROSAT and the latter given by the spacecraft clock. In this time-direction coordinate system, the observatory's optical axis is commanded to perform a prescribed nominal attitude motion $a_{0}(t)$, i.e. to maintain a nominal pointing direction $p_{0}(t)$ of the optical axis and a nominal roll angle $\rho_{0}(t)$ in the observation interval $\boldsymbol{T}$,

$a_{0}(t):=\left(p_{0}(t), \rho_{0}(t)\right), \quad p_{0}(t) \in S^{2}, \quad \rho_{0}(t) \in[0,2 \pi)$.

\footnotetext{
3 The expressions $A:=B$ or $B=: A$ define $A$ by $B$.
}

The nominal roll angle $\rho_{0}(t)$ in Eq. (3) is measured in the plane perpendicular to the optical axis away from a reference direction in a reference sense. The observatory's attitude control loop responds with a stochastic attitude process $a(t)$ to the nominal attitude $a_{0}(t)$ and to unavoidable stochastical drag forces. It performs a jitter motion $\delta a(t)$ around the nominal attitude ${ }^{4}$,

$$
\begin{aligned}
& a(t)=:(p(t), \rho(t)), \\
& p(t)=: p_{0}(t)+\delta p(t), \boldsymbol{E}[p(t)]=p_{0}(t), \operatorname{Var}[p(t)] \leq \sigma_{p}^{2} \\
& \rho(t)=: \rho_{0}(t)+\delta \rho(t), \boldsymbol{E}[\rho(t)]=\rho_{0}(t), \operatorname{Var}[\rho(t)] \leq \sigma_{\rho}^{2}
\end{aligned}
$$

In the absolute UTC time frame, an uncertainty of $3 \mu \mathrm{s}$ is to be added. The satellite control center provides the bounds $\sigma_{p}, \sigma_{\rho}$. The time measurement is made with a spacecraft clock based on a stabilized quartz oscillator running at precision $\sigma_{t}=100 \mu \mathrm{s}$,

$t=: t_{0}+\delta t, \quad 0 \leq \delta t \leq \sigma_{t}$.

The registering detectors in the focal plane have finite resolutions with respect to detector location $(X, Y)$, photon energy (measured via signal amplitude $A$ ), and photon arrival time $t$. Thus the measured quantities can be represented by an exact indexed quantity and a $\delta$ error term,

$X=: X_{0}+\delta X, \quad Y=: Y_{0}+\delta Y$,

$A=: A_{0}+\delta A, \quad|\delta X|,|\delta Y| \leq \sigma_{X}=\sigma_{Y},|\delta A| \leq \sigma_{A}$.

Here, $\sigma_{X}, \sigma_{Y}$ are the common linear pixel sizes expressed in angular units. $\sigma_{A}$ is the detector channel width, see Table 2. The true quantities are not observable but are estimable.

\subsection{Modelling errors}

Classical parameter estimation compares the data from an existing but unknown population with data from a hypothetical but known population. To make a comparison possible, the generation process for both kinds of data should be the same. This requires a mathematical model of the observatory. Therefore, much work was dedicated to the on-ground measurements and in-orbit calibrations of the observatory's PSFs.

For the ROSAT observatory, the PSF is factorized into two factors, a relative efficiency factor, $V(A, \epsilon)$ and the local PSF density. The first factor is termed the vignetting factor. It has the on-axis normalization $V_{0}(A, 0)=1$ and depends on the detector amplitude $A$ as well as on the off-axis angle $\epsilon$. The PSF density at point $(X, Y)$ for given source position $\left(X_{\mathrm{S}}, Y_{\mathrm{S}}\right)$ depends on the source amplitude $A_{\mathrm{S}}$, and source extension parameter $v_{\mathrm{S}}$

$$
\begin{aligned}
& p_{0}^{V}\left(X, Y \mid X_{\mathrm{S}}, Y_{\mathrm{S}}, A_{\mathrm{S}}\right):= V_{0}\left(A_{\mathrm{S}}, \epsilon_{\mathrm{S}}\right) \cdot \\
& p_{0}\left(X, Y \mid X_{\mathrm{S}}, Y_{\mathrm{S}}, A_{\mathrm{S}}\right), \\
& \int_{\boldsymbol{R}^{2}} p_{0}\left(X, Y \mid X_{\mathrm{S}}, Y_{\mathrm{S}}, A_{\mathrm{S}}, \epsilon_{\mathrm{S}}\right) \mathrm{d} X \mathrm{~d} Y=1
\end{aligned}
$$

Figure 3 shows the observed ROSAT PSPC point spread function at a large off-axis angle $\epsilon=44.2$ arcmin. The 16 rays emanating from the centre are due to the mechanical support struts

\footnotetext{
${ }^{4} \boldsymbol{E}[\boldsymbol{X}]$ denotes the expected value of the random variable $\boldsymbol{X}$ and $\operatorname{Var}[\boldsymbol{X}]$ is the variance of $\boldsymbol{X}$.
} 


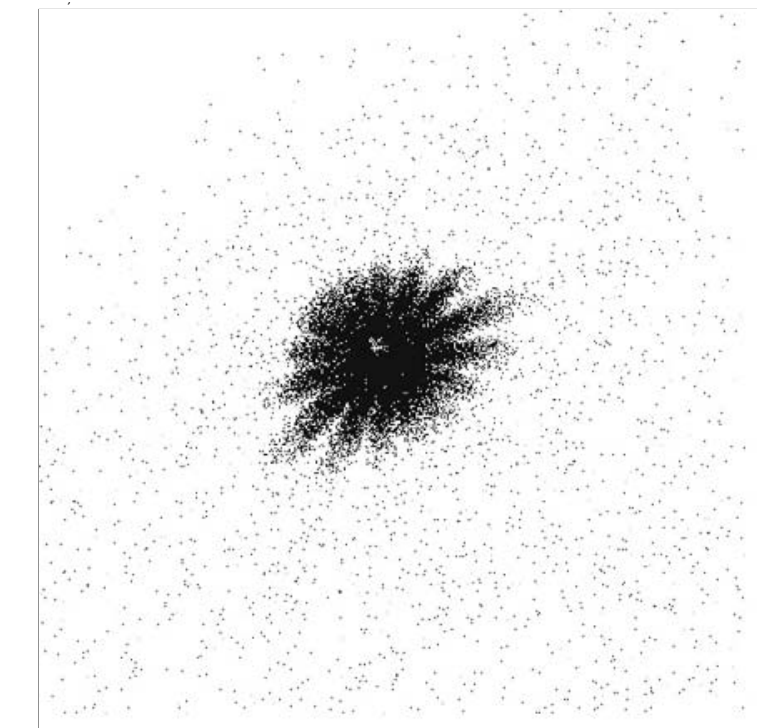

Fig. 3. The measured ROSAT PSPC PSF density at a large off-axis angle $\epsilon=44.2$ arcmin. At smaller off-axis angles the PSF is to a good approximation circularly symmetric Gaussian.

of the telescope, see Pfeffermann et al. (2003). The white spot at the centre indicates an undesirable local depression of the PSF density. Only the azimuthally averaged part of the PSF of Fig. 3 has been parameterized and estimated from calibration observations, see Paper I. For reasons of computational speed a further simplification was accepted. The best fit PSF density in radially symmetric Gaussian form (see formula (7) in Paper I) is taken as the PSF model in SASS and EXSAS for source estimation. The vignetting factor is only known to a limited precision. Also, the gas detectors age with time, cf. Pfeffermann et al. (2003). So, we account for the uncertainties in the PSF and vignetting factor by adopting the true-plusperturbation splitting

$$
\begin{aligned}
V(A, \epsilon)=: & V_{0}(A, \epsilon)+\delta V(A, \epsilon) \\
p\left(X, Y \mid X_{\mathrm{S}}, Y_{\mathrm{S}}, A_{\mathrm{S}}\right)=: & p_{0}\left(X, Y \mid X_{\mathrm{S}}, Y_{\mathrm{S}}, A_{\mathrm{S}}\right) \\
+ & \delta p\left(X, Y \mid X_{\mathrm{S}}, Y_{\mathrm{S}}, A_{\mathrm{S}}\right) .
\end{aligned}
$$

A deeply rooted modelling step concerns the photon source. The infall of the photons is a Poisson process in time $t$, celestial direction $(X, Y)$, and detector amplitude $A$ with a local mean count rate $\lambda(t, X, Y, A)$ counts/( $\operatorname{sarcsec}^{2}$ channel). This means: (a) the statistical independence of the counts from disjoint regions in the $(t, X, Y, A)$ space and (b) the Poisson distribution ${ }^{5}$

$$
\begin{aligned}
p_{0}(n \mid \lambda): & =\operatorname{Prob}[N=n], \\
& =\mathrm{e}^{-\lambda(t, X, Y, A)} \lambda(t, X, Y, A)^{n} / n !
\end{aligned}
$$

for the random number $N$ of observed photons. The observed distribution $p(n \mid \lambda)$ may deviate from the Poisson model by $\delta p(n \mid \lambda)$. So, again the decomposition

$p(n \mid \lambda)=: p_{0}(n \mid \lambda)+\delta p(n)$.

In Sect. 7 some light is shed on the distribution deviation $\delta p(n)$. The background estimator BGE is not error-free. For example

\footnotetext{
${ }^{5}$ Capital bold letters stand for random variables.
}

the background estimate is not count preserving. The background smoothness is also questionable. This dictates the introduction of a background deviation $\delta \lambda_{\mathrm{B}}(X, Y)$ from the exact, unknown background $\lambda_{\mathrm{B}, 0}(X, Y)$,

$\lambda_{\mathrm{B}}(X, Y)=: \lambda_{\mathrm{B}, 0}(X, Y)+\delta \lambda_{\mathrm{B}}(X, Y)$.

\subsection{Methodological errors}

In the passage from left to right in the block SAN in Fig. 2, equations of the form $F(x)=0$ for certain functions $F$ and unior multivariate arguments $x$ are to be solved. This is done by a numerical method. The convergence of the method is not always theoretically supported. One trusts only sensible results. The calculations are stopped when some termination criterion is reached. Therefore, one obtains approximate solutions $x$ deviating by $\delta x$ from the true solution $x_{0}$,

$x=: x_{0}+\delta x$.

Here, these errors $\delta x$ are called methodological. We shall now study the concrete appearance of the errors $\delta x$ from Eq. (12) in the system from Fig. 2.

We denote by $L[p \mid \mathcal{P}]$ the log-likelihood for the source parameter vector $p$ to have caused the photon sample $\mathcal{P}$. The maximizer $\hat{p}$ in the interior of the parameter space $\boldsymbol{P}$ of

$L[\mathcal{P}]:=\max _{p \in \boldsymbol{P}} L[p \mid \mathcal{P}]$

is found approximately by solving iteratively the necessary conditions. This is the vanishing gradient of the log-likelihood with respect to the source parameter vector,

$\frac{\partial L[p \mid \mathcal{P}]}{\partial p}=0$

The fixed point iteration, see Paper II, is stopped when with chosen tolerances $\sigma_{L, 1}>0$ to $\sigma_{L, 4}>0$ the termination criterion

$\left|\frac{\partial L[p \mid \mathcal{P}]}{\partial p_{k}}\right| \leq \sigma_{L, k}, \quad k=1,2,3,4$,

is reached. In principle, methodological errors can be reduced to a negligible size compared to others. In practice, however, this is not always the case.

All the errors from the different classes from Eq. (2) discussed so far contribute to a deviation $\delta p$ from the true unknown source parameter vector $p_{0}$,

$\hat{p}=: p_{0}+\delta \hat{p}$.

\subsection{The uncertainties of the statistical errors}

Strictly speaking, an estimate $\hat{p}$ for a parameter $p$ without a probabilistic statement on its reliability is useless. There are several ways to assign statistical errors to parameter estimates. Classical error assignments are (a) standard errors, and (b) confidence intervals. Also bootstrap errors, see Efron (1993), or the error treatment as in Bayesian statistics in which (prior) distributions are to be assigned to parameters location ${ }^{6}$, come

\footnotetext{
${ }^{6}$ Random variables possess distributions. Their interpretation in both cases is radically different.
} 
into consideration, cf. O’Hagan (1994), Babu \& Feigelson (1992, 1999), especially the contribution by T. Loredo.

In SASS and EXSAS standard errors are calculated. They are based on the normal distribution. For distributions $F_{\boldsymbol{X}}$ of a random variable $\boldsymbol{X}$ which are far from normality, standard errors, i.e. $\sqrt{\operatorname{Var}[\boldsymbol{X}]}$, are senseless. Fortunately, for large samples, many, but not all ${ }^{7}$, statistics tend to normality due to the effect of the central limit theorem.

The infalling random number $N$ of observed photons from the random direction $(\boldsymbol{X}, \boldsymbol{Y})$ causing a random detector amplitude $\boldsymbol{A}$ in the detector leads to a random source parameter $\boldsymbol{p}$ from Eq. (1) in the output list $\mathcal{S}$ in Fig. 2. As such, the source parameter has a sample distribution density $f_{p}^{N}(\Lambda, X, Y, A)$ for any sample size $N>0$. Unfortunately, in our case, this distribution is hard to access because $\boldsymbol{p}$ is an implicitly defined function of many random variables. The problem becomes more accessible in the passage to large samples $N \rightarrow \infty$. The limiting distribution $f_{p}^{\infty}(\Lambda, X, Y, A)$ is known to be normal. Calculating standard errors based on incorrect distributions introduces deviations $\delta$ from the true standard errors,

$$
\begin{aligned}
\sigma_{X_{\mathrm{S}}}=: \sigma_{X_{\mathrm{S}}, 0}+\delta \sigma_{X_{\mathrm{S}}}, & \sigma_{Y_{\mathrm{S}}}=: \sigma_{Y_{\mathrm{S}}, 0}+\delta \sigma_{Y_{\mathrm{S}}}, \\
\sigma_{\lambda_{\mathrm{S}}}=: \sigma_{\lambda_{\mathrm{S}}, 0}+\delta \sigma_{\lambda_{\mathrm{S}}}, & \sigma_{\nu_{\mathrm{S}}}=: \sigma_{v_{\mathrm{S}}, 0}+\delta \sigma_{\nu_{\mathrm{S}}} .
\end{aligned}
$$

We are faced with a design dilemma already discussed in Paper II: The MLE box in Fig. 2 is tailored for faint X-ray sources. In contrast, the error assignment in the same box needs strong sources. Thus, the errors for the source parameters are expected to become unreliable more quickly than the parameter estimates themselves as the sources become weaker and weaker.

\subsection{Concepts for the perturbation analysis}

Now, all error sources are identified. We do not discuss methodological errors. Table 2 gives a summary of the remaining error sources and the number of components $C$ for them, and their tolerances when available or applicable.

We may now define the nominal design vector $q_{0}$, already introduced in Sect. 2, its variation $\delta q$, and the perturbed design vector $q$ by

$$
\begin{aligned}
q:= & q_{0}+\delta q, \\
q_{0}:= & \left(p_{0}(t), \rho_{0}(t), t_{0}, X_{0}, Y_{0}, A_{0}, V_{0}(A, \epsilon),\right. \\
& \left.p_{0}(n \mid \lambda), p_{0}(X, Y), \lambda_{\mathrm{B}, 0}(X, Y)\right), \\
\delta q:= & (\delta p(t), \delta \rho(t), \delta t, \delta X, \delta Y, \delta A, \delta V(A, \epsilon), \\
& \left.\delta p(n \mid \lambda), \delta p(X, Y), \delta \lambda_{\mathrm{B}}(X, Y)\right) .
\end{aligned}
$$

Notice that $\delta q$ contains different quantities: ordinary parameters $\delta t, \delta X, \delta Y$, random variables or stochastic processes $\delta p(t), \delta \rho(t)$, and functions $\delta \lambda_{\mathrm{B}}(X, Y), \delta p(X, Y)$. Consider for instance the nominal Poisson distribution $p_{0}(n \mid \lambda)$. This means the system is designed to process photons which follow a Poisson distribution. We vary this assumption when we consider the system performance under the perturbed stochastic photon process $p(n \mid \lambda):=p_{0}(n \mid \lambda)+\delta p(n \mid \lambda)$. All variations are assumed to be mutually independent.

\footnotetext{
${ }^{7}$ Extreme value statistics do not tend to normality.
}

Table 2. The ROSAT error sources with number $C$ of components, symbols for perturbations, and tolerances $\sigma$ or relative errors. The $\sigma$ are based on measured attitude time series, clock frequency of the onbord timer, linear sizes of the detector pixels, PSF and background measurements, and statistical test.

\begin{tabular}{llll}
\hline \hline Error source & $C$ & Symbol & $\sigma$ \\
\hline Satellite attitude & 2 & $\delta p(t), \delta \rho(t)$ & $6^{\prime \prime}, 1^{\circ}$ \\
Time measurement & 1 & $\delta t$ & $100 \mu \mathrm{s}$ \\
Detector location & 2 & $\delta X, \delta Y$ & $0.25^{\prime \prime}, 0.25^{\prime \prime}$ \\
Detector amplitude & 1 & $\delta A$ & $\sqrt{A} / 256$ \\
PSF & 1 & $\delta \pi(X, Y)$ & $10 \%$ \\
Background & 1 & $\delta \lambda_{\mathrm{B}}(X, Y)$ & $10 \%$ \\
Poisson distribution & 1 & $\delta p(n)$ & $15 \%$ \\
\hline
\end{tabular}

A variational analysis supplies us with forward perturbation results for the system considered with respect to the nominal design vector $q_{0}$. "Forward" means: in the direction of the arrow in Fig. 2. What is the effect of the joint independent variations of all component quantities of $\delta q$ from Eq. (18) on the four source parameter components of $p$ ?

Two approaches to find out the robustness properties of the system from Fig. 2 are discussed in Sect. 6: (a) an analytical treatment and (b) variations of the $\delta q$-quantities in simulations. Section 4 reports on the simulation results. A combination of both is desirable, as the discussion in Sect. 7 will show. To our knowledge, no rigorous analytical treatment exists. Source estimation with respect to PSF variation $\delta p\left(X, Y \mid X_{\mathrm{S}}, Y_{\mathrm{S}}, A_{\mathrm{S}}\right)$ and background variation $\delta \lambda_{\mathrm{B}}(X, Y)$ is fundamental in larger parts of observational astronomy and deserves a formal analytic treatment too. See Jaschek \& Murtagh (1990) for questions on uncertainties, small samples, and errors similar to ours.

\section{The analytic perturbation analysis}

The $\log$-likelihood $L$ in dependence on the source parameter $p$ vector is a function of several real variables, Hence, multivariate calculus applies. The gradient of $L$ with respect to the parameter vector $p$ is designated by $L_{p}$. Similarly, $L_{p, p}$ means the Hessian of $L$, the matrix of the second order partial derivatives. For the ROSAT log-likelihood, the Hessian $L_{p, p}$ was quoted elementwise in Paper II.

The $\log$-likelihood $L$ in its dependence on the design vector $q$ is a functional, i.e. depends also on functions. As we wish to study the change of the log-likelihood with respect to the design vector $q$, the variational derivative, $L_{q}$, of $L$, a linear operator, gains in importance here replacing the usual derivative from calculus. We are to use the calculus in complete normed spaces. The observed photon sample $\mathcal{P}$ is kept fixed. Thus, the subscript $q$ in Eqs. (19) to (23) stands for a variational derivative $^{8}$. In this notation, $L_{p, q}$ is the variational derivative of the gradient.

\footnotetext{
8 The definition of the derivative, $F^{\prime}[x]$, of a map $F[x]$ between two normed spaces is $F[x+h]-F[x]-F^{\prime}[x] h=o(h)$ for $h \rightarrow 0$.
} 


\subsection{Likelihood dependence on the design vector}

The first major step is to establish the log-likelihood functional $L[p \mid \mathcal{P}]$ explicitly in its dependence on the design vector $q$ as $L[p, q \mid \mathcal{P}]$. This step is to a certain degree instrumentdependent and depends on the adopted mathematical model for it. No attempts to formulate a canonical model for the general class are made here.

\subsection{General linear perturbation analysis}

Assume the vectors $p, q$ are defined, possibly different from Eqs. (1) or (18). Having a log-likelihood $L[p, q \mid \mathcal{P}]$, we are able to calculate the variations of interest. We assume the differentiability of $L$ with respect to both arguments $p, q$ in this section. Of interest are (a) the likelihood variation $\delta L[p, q \mid \mathcal{P}]$ taken at the log-likelihood maximizer $\hat{p}$ and the nominal design vector $q:=q_{0}$, and (b) the shift $\delta p:=\delta p[\delta q]$ of the maximizer $p:=\hat{p}$ of $L[p, q \mid \mathcal{P}]$ due to the variation $\delta q$ of the design vector which vanishes in the undisturbed case, $\delta p[0]:=0$.

We begin with the likelihood variation in linear approximation for small perturbations,

$$
\begin{aligned}
\delta L\left[\hat{p}, q_{0} \mid \mathcal{P}\right]: & =L\left[\hat{p}+\delta p, q_{0}+\delta q \mid \mathcal{P}\right]-L\left[\hat{p}, q_{0} \mid \mathcal{P}\right], \\
& =L_{q}\left[\hat{p}, q_{0} \mid \mathcal{P}\right] \delta q+O\left(\|\delta q\|^{2}\right) .
\end{aligned}
$$

The vanishing gradient $L_{p}\left[\hat{p}, q_{0}\right]=0$ is used in Eq. (19).

The shift $\delta p$ of the source parameter vector is astronomically more important. We need a representation of an implicitly defined map in terms of the defining map - well-known in the case of functions instead of mappings, cf. the example in Sect. 7.

According to Eq. (14), at the maximizer $\hat{p}$ of $L$ its gradient $L_{p}$ vanishes. We assume the existence of the differentiable mapping $\delta p[\delta q]$ with derivative $\delta p / \delta q$. Forming the partial derivatives and using the chain rule in the identity in $\delta q$ in the first line of Eq. (20) leads us to the second line. The variations $\delta q$ are arbitrary and the braced expression must vanish because the error term is of a higher order for $\delta q \rightarrow 0$. We have the derivative in $\delta p / \delta q$ terms of the log-likelihood in the third line,

$$
\begin{aligned}
0 & =L_{p}\left[\hat{p}+\delta p[\delta q], q_{0}+\delta q \mid \mathcal{P}\right], \\
& =\left\{L_{p, p}\left[\hat{p}, q_{0} \mid \mathcal{P}\right] \frac{\delta p}{\delta q}+L_{p, q}\left[\hat{p}, q_{0} \mid \mathcal{P}\right]\right\} \delta q+O\left(\|\delta q\|^{2}\right), \\
\frac{\delta p}{\delta q} & :=-L_{p, p}^{-1}\left[\hat{p}, q_{0}\right] L_{p, q}\left[\hat{p}, q_{0}\right] .
\end{aligned}
$$

How the last formula works in the case of functions is illustrated by a simple example in Sect. 7. To ascertain the existence of the differentiable mapping $\delta p[\delta q]$, we invoke the Implicit Mapping Theorem, cf. Lang (1983, p. 131). For this purpose, the log-likelihood gradient map

$L_{p}[p, q \mid \mathcal{P}]: \boldsymbol{P} \times \boldsymbol{Q} \rightarrow \boldsymbol{R}^{d}, \quad d:=\operatorname{dim}(\boldsymbol{P})$,

is to be considered between the three complete normed spaces $\boldsymbol{P}, \boldsymbol{Q}, \boldsymbol{R}^{d}$, each one equipped with an appropriate norm $\|\cdot\|$. The latter can be assigned in many ways - according to the astronomer's error valuation. The norm in $\boldsymbol{Q}$ is required by Eq. (19). Without a norm, one cannot decide whether a perturbation $\delta q$ is small or not. The invertibility condition $\operatorname{det}\left(L_{p, p}\left[\hat{p}, q_{0} \mid \mathcal{P}\right]\right) \neq 0$ guarantees the the mapping $\delta p[\delta q]$ being of the same smoothness as $L_{p}$ for sufficiently small perturbations $\|\delta q\| \leq \rho,(\rho>0$ small $)$. So, the assumed differentiability of $L_{q}$ carries over to $\delta p / \delta q$. By continuity of $\delta p[\delta q]$ in $\delta q$, inferred from its differentiability, $\delta p$ stays small for small perturbations $\delta q$, and the remainder term is negligible.

We state the main result for the MLE perturbation from Eq. (20) formally.

\section{Lemma 1 (Linear perturbation of ML source estimates).}

Let $L[p, q \mid \mathcal{P}]$ be the log-likelihood for the source vector $p$ to have caused the X-ray photon sample $\mathcal{P}$ under the design vector $q$. Let $q_{0}$ be the nominal design vector of the observatory-analyzer system subject to the variation $\delta q$. Denote by $\hat{p}$ the maximizer of the log-likelihood for the nominal design vector $q_{0}$. Then the variations of the log-likelihood $L$ and of the source parameter vector $p$ are given in a linear approximation by

$$
\begin{aligned}
\delta L[p, q \mid \mathcal{P}] & :=L_{q}\left[\hat{p}, q_{0} \mid \mathcal{P}\right] \delta q+O\left(\|\delta q\|^{2}\right), \\
\delta p & =\frac{\delta p}{\delta q} \delta q+O\left(\|\delta q\|^{2}\right) \\
\frac{\delta p}{\delta q} & :=-L_{p, p}^{-1}\left[\hat{p}, q_{0}\right] L_{p, q}\left[\hat{p}, q_{0}\right] .
\end{aligned}
$$

To evaluate the variations $\delta L$ and $\delta p$ in linear approximation from Eq. (22), we need to know the three linear operators $L_{p, p}, L_{q}, L_{p, q}$, all three evaluated at $\left(\hat{p}, q_{0}\right)$ and the last two operating on the perturbation $\delta q$.

The linear part of the source parameter shift $\delta p$ due to the perturbation $\delta q$ is explicitly calculable in terms of second derivatives of the log-likelihood, namely the inverse of the Hessian and the variational derivative of the gradient evaluated at the maximizer $\hat{p}$ and the nominal design vector $q_{0}$. An immediate consequence is the estimate in terms of matrix norms

$$
\begin{aligned}
\|\delta p\| & =\left\|\frac{\delta p}{\delta q} \delta q\right\|+O\left(\|\delta q\|^{2}\right), \\
& \leq\left\|L_{p, p}^{-1}\left[\hat{p}, q_{0} \mid \mathcal{P}\right]\right\| \cdot\left\|L_{p, q}\left[\hat{p}, q_{0} \mid \mathcal{P}\right] \delta q\right\|+O\left(\|\delta q\|^{2}\right) .
\end{aligned}
$$

The remaining problem is to choose and evaluate the matrix norms at the (unknown) maximizer $\hat{p}$ for the nominal design vector $q_{0}$. This will be developed further in the case of the ROSAT log-likelihood and for a two-component design vector $q$ to follow.

\subsection{Likelihood perturbation with respect to background and PSF}

In a modest but practically important scenario, only perturbations $\delta q:=\left(\delta \lambda_{\mathrm{B}}, \delta p\right)$ with respect to background density $\lambda_{\mathrm{B}}(t, X, Y, A)$ and PSF density $\pi(X, Y):=$ $p\left(X, Y \mid X_{\mathrm{S}}, Y_{\mathrm{S}}, v_{\mathrm{S}}, A_{\mathrm{S}}\right)$ will be considered. The perturbation $\delta q$ is permissible as long as the total count density $\lambda:=\lambda_{\mathrm{B}}+\lambda_{\mathrm{S}} V p$ stays pointwise positive, $\lambda>0$. 
We depart from the log-likelihood functional $L\left[p, \lambda_{\mathrm{B}}, \pi \mid \mathcal{P}\right]$ as given in Paper II in a notation adapted to the present purposes. The dependence on the source parameter vector $p$ is suppressed; the dependence on the densities $\lambda_{\mathrm{B}}, \pi$ is stressed,

$$
\begin{aligned}
L\left[p, \lambda_{\mathrm{B}}, \pi \mid \mathcal{P}\right] & :=-\Lambda_{\mathrm{B}}-\lambda_{\mathrm{S}} T^{V}+\sum_{m=1}^{M} \ln \left[\lambda_{\mathrm{B}, m}+\lambda_{\mathrm{S}} p_{m}^{V}\right], \\
\lambda_{\mathrm{B}, m} & :=\lambda_{\mathrm{B}}\left(t_{m}, X_{m}, Y_{m}, A_{m}\right), \\
p_{m}^{V} & :=V\left[A_{m}, \epsilon\left(x_{m}, y_{m}\right)\right] \cdot \pi\left(X_{m}, Y_{m}\right), \\
\pi(X, Y) & :=p\left(X, Y \mid X_{\mathrm{S}}, Y_{\mathrm{S}}, \nu_{\mathrm{S}}, A_{\mathrm{S}}\right) .
\end{aligned}
$$

The point $\left(x_{m}, y_{m}\right)$ is the impact point of the $m$ th photon in the detector plane in detector coordinates. We form the likelihood variation $\delta L\left[p, \lambda_{\mathrm{B}}, \pi \mid \mathcal{P}\right]\left(\delta \lambda_{\mathrm{B}}, \delta p\right)$ along with the likelihood differential $L_{\lambda}\left[p, \lambda_{\mathrm{B}}, \pi \mid \mathcal{P}\right] \delta \lambda$ and obtain

$$
\begin{aligned}
\delta L\left[p, \lambda_{\mathrm{B}}, \pi \mid \mathcal{P}\right]\left(\delta \lambda_{\mathrm{B}}, \delta p\right) & =-\delta \Lambda_{\mathrm{B}}+\sum_{m=1}^{M} \ln \left[1+\frac{\delta \lambda_{m}}{\lambda_{m}}\right], \\
L_{\lambda}\left[p, \lambda_{\mathrm{B}}, \pi \mid \mathcal{P}\right] \delta \lambda & =-\delta \Lambda_{\mathrm{B}}+\sum_{m=1}^{M} \frac{\delta \lambda_{m}}{\lambda_{m}}, \\
\lambda_{m} & :=\lambda_{\mathrm{B}, m}+\lambda_{\mathrm{S}} p_{m}^{V}, \\
\delta \lambda_{m} & :=\delta \lambda_{\mathrm{B}, m}+\lambda_{\mathrm{S}} V_{m} \delta p_{m} .
\end{aligned}
$$

The admissibility condition $\lambda>0$ is satisfied in the class $\Pi\left(\sigma_{\lambda}\right)$ of perturbations $\delta q$ with the relative size parameter $\sigma_{\lambda}$. It contains for all $\lambda_{\mathrm{S}} \geq 0$ the subclass $\Pi^{\prime}\left(\sigma_{\lambda}\right)$,

$$
\begin{aligned}
\Pi\left(\sigma_{\lambda}\right): & \frac{\left|\delta \lambda_{\mathrm{B}}+\lambda_{\mathrm{S}} V \delta p\right|}{\lambda_{\mathrm{B}}+\lambda_{\mathrm{S}} V p} \leq \sigma_{\lambda}, \quad 0 \leq \sigma_{\lambda}<1, \\
\Pi^{\prime}\left(\sigma_{\lambda}\right): & \frac{\left|\delta \lambda_{\mathrm{B}}\right|}{\lambda_{\mathrm{B}}} \leq \sigma_{\lambda}, \quad \frac{|\delta p|}{p} \leq \sigma_{\lambda} .
\end{aligned}
$$

The perturbation class $\Pi^{\prime}\left(\sigma_{\lambda}\right)$ is one-parametric; the $\sigma$ in both occurrences in Eq. (26) are the same.

The next lemma provides a nonlinear non-statisical forward upper bound and backward lower bound perturbation result for not necessarily small perturbations in background and PSF.

Lemma 2 (Log-likelihood perturbation). Let $\delta q:=\left(\delta \lambda_{\mathrm{B}}\right.$, $\delta p)$ be a joint background and PSF perturbation from $E q$. (26) with relative size parameter $\sigma_{\lambda}<1$. Then the log-likelihood perturbation $\delta L$ is bounded in magnitude by

$$
\begin{aligned}
|\delta L| & \leq \overline{\delta L}, \\
\overline{\delta L} & :=\left|\delta \Lambda_{\mathrm{B}}\right|-\ln \left(1-\sigma_{\lambda}\right) .
\end{aligned}
$$

Conversely, if a log-likelihood uncertainty of size $\overline{\delta L}$ is tolerated, to all perturbations respecting this tolerance belongs the perturbation class $\Pi^{\prime}\left(\bar{\sigma}_{\lambda}\right)$ from Eq. (26) i.e.

$$
\begin{aligned}
& \sigma_{\lambda} \leq \bar{\sigma}_{\lambda}:=1-\mathrm{e}^{-\left(\overline{\delta L}-\left|\delta \Lambda_{\mathrm{B}}\right|\right) / M}, \quad \overline{\delta L} \geq \delta \Lambda_{\mathrm{B}}, \\
& \sigma_{\lambda}:=\max \left\{\frac{\left|\delta \lambda_{\mathrm{B}}\right|}{\lambda_{\mathrm{B}}}, \frac{|\delta p|}{p}\right\} .
\end{aligned}
$$

Proof. It may suffice to show the main inequality, the estimation of $\delta \lambda_{m}$ in terms of $\lambda_{m}$, uniformly in $\lambda_{\mathrm{S}}$,

$$
\begin{aligned}
\left|\delta \lambda_{m}\right| & :=\left|\delta \lambda_{\mathrm{B}, m}+\lambda_{\mathrm{S}} V_{m} \delta p_{m}\right|, \\
& \leq \lambda_{m} \cdot \max \left\{\frac{\left|\delta \lambda_{\mathrm{B}, m}\right|}{\lambda_{\mathrm{B}, m}}, \frac{\left|\delta p_{m}\right|}{p_{m}}\right\} .
\end{aligned}
$$

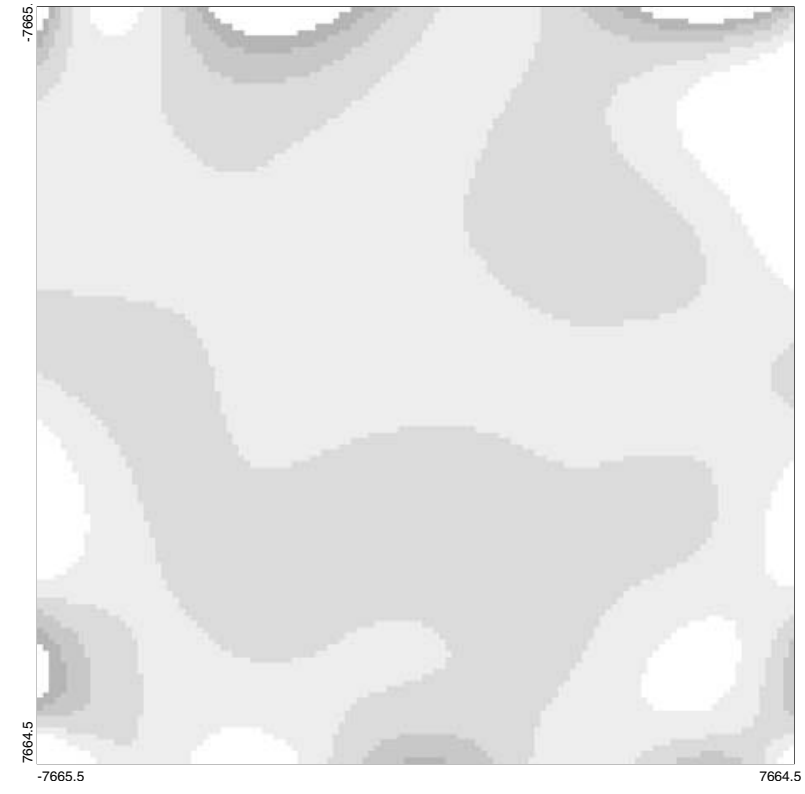

Fig. 4. A background estimate $\hat{\lambda}_{\mathrm{B}}$ for a nominal uniform background $\lambda_{\mathrm{B}}=0.009 \mathrm{cts} / \mathrm{sky} \_$pix ${ }^{2}$ (dark $=$ high values). The range is $10^{-4} \leq$ $\hat{\lambda}_{\mathrm{B}} \leq 0.0257$. The mean, $\hat{\mu}_{\lambda_{\mathrm{B}}}=0.00855$, is $-5.6 \%$ away from the true value. The standard deviation, $\hat{\sigma}_{\lambda_{\mathrm{B}}}=0.00355$, is about $40 \%$ of the mean. The estimated number of background photons, $\hat{\Lambda}_{\mathrm{B}}=2241.7$, underestimates the true value by $5 \%$.

Observe the logarithmical divergence $\overline{\delta L} \rightarrow \infty$ for $\sigma_{\lambda} \rightarrow 1$. The $\log$-likelihood $L$ may assume any real value, positive or negative. The likelihood is an almost linear function of the sample size $M$, i.e. there are constants $\underline{c}(\mathcal{P})<\bar{c}(\mathcal{P})$ such that $\underline{c}(\mathcal{P}) \leq L / M \leq \bar{c}(\mathcal{P})$. This means for the relative likelihood error the approximate relation $|\delta L / L| \approx-\ln \left(1-\sigma_{\lambda}\right)$. Inequality 28 tells us how precisely both the background and PSF perturbations must be modelled in order not to exceed a prescribed length $\overline{\delta L}$ of the likelihood error bar.

Example. Assume only count-preserving background perturbations, $\delta \Lambda_{\mathrm{B}}=0$, are considered and about a $10 \%$ likelihood error is tolerable, i.e. $\overline{\delta L}=M / 10$. Then $\sigma_{\lambda}:=1-\mathrm{e}^{-0.1}>0.095$. The lemma assures us that up to $9.5 \%$ perturbations in background and PSF, mutually independent or not, are allowed for an arbitrary observed sample $\mathcal{P}$ of size $M$ and for any value $\lambda_{\mathrm{S}} \geq 0$ of the hypothesized source density parameter. For a tolerated $150 \%$ likelihood uncertainty instead of $10 \%$, the perturbation size rises to $77 \%$ by the lemma. It may be that still some perturbations larger than $9.5 \%$ do not exceed the $10 \%$ tolerance - but this is not true for all perturbations.

\subsection{Perturbation with respect to background}

Figure 4 shows an estimate $\hat{\lambda}_{\mathrm{B}}$ from BGE for a nominal uniform density $\lambda_{\mathrm{B}}=0.009 \mathrm{cts} / \mathrm{sky} \_$pix ${ }^{2}$ which means $\Lambda_{\mathrm{B}}=2359$ background photons for the PSPC carrying a $5 \%$ error. We see an unjustified oscillatory behaviour and steep slopes towards the margin stemming from the implemented class of bivariate splines. 
We start from the gradient $L_{p}[p, q \mid \mathcal{P}]:=\left(L_{X_{s}}, L_{Y_{s}}\right.$, $L_{\lambda_{s}}, L_{\nu_{s}}$ ) with four components taken from Paper II,

$$
\begin{aligned}
L_{X_{\mathrm{S}}}:= & 2 \lambda_{\mathrm{S}} \sum_{m=1}^{M} \frac{p_{m}^{V}}{\lambda_{\mathrm{B}, m}+\lambda_{\mathrm{S}} p_{m}^{V}} \cdot \frac{X_{\mathrm{S}}-X_{m}}{v_{m}}, \\
L_{Y_{\mathrm{S}}}:= & 2 \lambda_{\mathrm{S}} \sum_{m=1}^{M} \frac{p_{m}^{V}}{\lambda_{\mathrm{B}, m}+\lambda_{\mathrm{S}} p_{m}^{V}} \cdot \frac{Y_{\mathrm{S}}-Y_{m}}{v_{m}}, \\
L_{\lambda_{\mathrm{S}}}:= & -T^{V}+\sum_{m=1}^{M} \frac{p_{m}^{V}}{\lambda_{\mathrm{B}, m}+\lambda_{\mathrm{S}} p_{m}^{V}}, \\
L_{\gamma_{\mathrm{S}}}:= & \lambda_{\mathrm{S}} \cdot \sum_{m=1}^{M} \frac{p_{m}^{V}}{\lambda_{\mathrm{B}, m}+\lambda_{\mathrm{S}} p_{m}^{V}} \\
& \times\left[\frac{\left(X_{\mathrm{S}}-X_{m}\right)^{2}+\left(Y_{\mathrm{S}}-Y_{m}\right)^{2}}{v_{m}^{2}}-\frac{1}{v_{m}}\right], \\
p_{m}^{V}:= & V_{m} p_{m}\left(X_{\mathrm{S}}, Y_{\mathrm{S}}, v_{\mathrm{S}}\right) .
\end{aligned}
$$

Forming the derivatives according to the definition leads from Eq. (30) to the variational differentials $L_{p, q} \delta \lambda_{\mathrm{B}}$ in terms of the weights $w_{m}$ to

$$
\begin{aligned}
L_{X_{\mathrm{S}}, \lambda_{\mathrm{B}}} \delta \lambda_{\mathrm{B}}:= & -2 \lambda_{\mathrm{S}} \sum_{m=1}^{M} w_{m} \cdot \frac{X_{\mathrm{S}}-X_{m}}{v_{m}} \cdot \delta \lambda_{\mathrm{B}, m}, \\
L_{Y_{\mathrm{S}}, \lambda_{\mathrm{B}}} \delta \lambda_{\mathrm{B}}:= & -2 \lambda_{\mathrm{S}} \sum_{m=1}^{M} w_{m} \cdot \frac{Y_{\mathrm{S}}-Y_{m}}{v_{m}} \cdot \delta \lambda_{\mathrm{B}, m}, \\
L_{\lambda_{\mathrm{S}}, \lambda_{\mathrm{B}}} \delta \lambda_{\mathrm{B}}:= & -\sum_{m=1}^{M} w_{m} \cdot \delta \lambda_{\mathrm{B}, m}, \\
L_{\gamma_{\mathrm{S}}, \lambda_{\mathrm{B}}} \delta \lambda_{\mathrm{B}}:= & -\lambda_{\mathrm{S}} \cdot \sum_{m=1}^{M} w_{m} \cdot \delta \lambda_{\mathrm{B}, m} \\
& \times\left[\frac{\left(X_{\mathrm{S}}-X_{m}\right)^{2}+\left(Y_{\mathrm{S}}-Y_{m}\right)^{2}}{v_{m}^{2}}-\frac{1}{v_{m}}\right], \\
w_{m}:= & \frac{p_{m}^{V}}{\left(\lambda_{\mathrm{B}, m}+\lambda_{\mathrm{S}} p_{m}^{V}\right)^{2}} .
\end{aligned}
$$

\subsection{Perturbation with respect to PSF}

We proceed analogously with the derivatives with respect to the PSF. We depart again from Eq. (26). The variational differentials $L_{p, q} \delta p$ turn out to have the redefined weights $w_{m}$

$$
\begin{aligned}
L_{X_{\mathrm{S}}, \lambda_{\mathrm{B}}} \delta p:= & -2 \lambda_{\mathrm{S}}^{2} \sum_{m=1}^{M} w_{m} \cdot \frac{X_{\mathrm{S}}-X_{m}}{v_{m}} \cdot \delta \lambda_{\mathrm{B}, m}, \\
L_{Y_{\mathrm{S}}, \lambda_{\mathrm{B}}} \delta p:= & -2 \lambda_{\mathrm{S}}^{2} \sum_{m=1}^{M} w_{m} \cdot \frac{Y_{\mathrm{S}}-Y_{m}}{v_{m}} \cdot \delta \lambda_{\mathrm{B}, m}, \\
L_{\lambda_{\mathrm{S}}, \lambda_{\mathrm{B}}} \delta p:= & -\lambda_{\mathrm{S}} \sum_{m=1}^{M} w_{m} \cdot \delta \lambda_{\mathrm{B}, m}, \\
L_{\gamma_{\mathrm{S}}, \lambda_{\mathrm{B}}} \delta p:= & -\lambda_{\mathrm{S}}^{2} \cdot \sum_{m=1}^{M} w_{m} \cdot \delta \lambda_{\mathrm{B}, m} \\
& \times\left[\frac{\left(X_{\mathrm{S}}-X_{m}\right)^{2}+\left(Y_{\mathrm{S}}-Y_{m}\right)^{2}}{v_{m}^{2}}-\frac{1}{v_{m}}\right], \\
w_{m}:= & \frac{V_{m} \cdot p_{m}^{V}}{\left(\lambda_{\mathrm{B}, m}+\lambda_{\mathrm{S}} p_{m}^{V}\right)^{2}} .
\end{aligned}
$$

\section{Simulated ROSAT observations}

We now replace the hardware observatory box OBS in Fig. 2 by a software counterpart. A pointed observation of a stationary single point source with $\Lambda_{\mathrm{S}}$ source photons embedded in a uniform, stationary background of density $\lambda_{\mathrm{B}}$ is chosen as input ( $\delta$-Dirac distribution),

$$
\begin{aligned}
a_{0}(t):= & \left(p_{0}(t), \rho_{0}(t)\right), \\
\lambda(t, X, Y, A):= & \Lambda_{\mathrm{S}} \cdot \delta\left(X-X_{\mathrm{S}}, Y-Y_{\mathrm{S}}, A-A_{\mathrm{S}}\right) \\
& +\lambda_{\mathrm{B}} \cdot \delta\left(A-A_{\mathrm{B}}\right) .
\end{aligned}
$$

Under the choice in Eq. (33), a simulated ROSAT photon sample $\mathcal{P}$ representing a single ROSAT point source with a known source parameter vector $p_{0}$ embedded in a known uniform background is generated. After MLE we know both the true $p_{0}$ and estimated source parameter vector $\hat{p}$. This enables us to form the true random errors for the vector $p$ and for the vector of its $1 \sigma$ standard deviations $\sigma_{p}$, i.e. at the $68.3 \%$ significance level,

$\hat{e}_{p}:=\hat{p}-p_{0}, \quad \hat{e}_{\sigma_{p}}:=\left|\hat{e}_{p}\right|-\sqrt{\frac{2}{\pi}} \cdot \hat{\sigma}_{p}$.

Repeating the generation of the sample $\mathcal{P}$ and the subsequent MLE $N$ times, we obtain two empirical sampling distributions $\hat{F}_{e_{p}}^{N}$ and $\hat{F}_{e_{\sigma_{p}}}^{N}$ for the errors $\hat{e}_{p}$ and for $\hat{e}_{\sigma_{p}}$ from Eq. (34), respectively, with respect to the various source parameters appearing in Eq. (33) spanning the parameter space of $\mathcal{P}$ along with additional parameters hidden from the user. Appropriate statistics summarizing the empirical distributions can be calculated. As usual, we choose for them the location and spread parameters. The error variable $\hat{e}_{\sigma_{p}}$ in Eq. (34) is defined so that $\boldsymbol{E}\left[e_{\sigma_{p}}\right]=0$ in the parent population whenever $e_{p}$ is normally distributed.

\subsection{The simulation parameter space}

The simulation parameter space $S$ is the Cartesian product of three factor spaces: single source space $S_{\mathrm{S}}$, background space $\boldsymbol{S}_{\mathrm{B}}$, and observatory's operation space $\boldsymbol{S}_{\mathrm{O}}$ where $\boldsymbol{F}_{D}, \boldsymbol{A}_{D}$ in Eq. (35) are the field of view and amplitude space of the detector $D$ in focus out of the set $\boldsymbol{D}$ of the available detectors ${ }^{9}$,

$$
\begin{aligned}
\boldsymbol{S} & :=\boldsymbol{S}_{\mathrm{S}} \times \boldsymbol{S}_{\mathrm{B}} \times \boldsymbol{S}_{\mathrm{O}}, \\
\boldsymbol{S}_{\mathrm{S}} & :=\left\{\left(\lambda_{\mathrm{S}}, X_{\mathrm{S}}, Y_{\mathrm{S}}, \nu_{\mathrm{S}}\right) \in \boldsymbol{R}_{+} \times \boldsymbol{F}_{D} \times \boldsymbol{R}_{+}\right\}, \\
\boldsymbol{S}_{\mathrm{B}} & :=\left\{\left(\lambda_{\mathrm{B}}, A_{\mathrm{B}}\right) \in \boldsymbol{R}_{+} \times \boldsymbol{A}_{D}\right\}, \\
\boldsymbol{S}_{\mathrm{O}} & :=\left\{\left(w_{\mathrm{A}}, w_{\mathrm{P}}, w_{\mathrm{R}}, D\right) \in \boldsymbol{R}_{+}^{2} \times[0,2 \pi] \times \boldsymbol{D}\right\} .
\end{aligned}
$$

According to Eq. (35), the dimension of $\boldsymbol{S}$ is $\operatorname{dim}(\boldsymbol{S})=10$. For a group of $s \geq 1$ sources the dimension of the joint parameter space $\boldsymbol{S}^{(s)}:=\boldsymbol{S}_{\mathrm{S}}^{s} \times \boldsymbol{S}_{\mathrm{B}} \times \boldsymbol{S}_{\mathrm{O}}$ is $\operatorname{dim}\left(\boldsymbol{S}^{(s)}\right)=4 s+6$. In Sect. 4.6 we shall briefly consider a pair, $s=2$, of confluent sources.

\subsection{Exploration of the parameter space}

Since the space $S$ is high-dimensional, an appropriate strategy to explore this parameter space needs to be found. We decide to explore the parameter space pathwise.

${ }^{9} \boldsymbol{R}_{+}$is the set of nonnegative real numbers. 

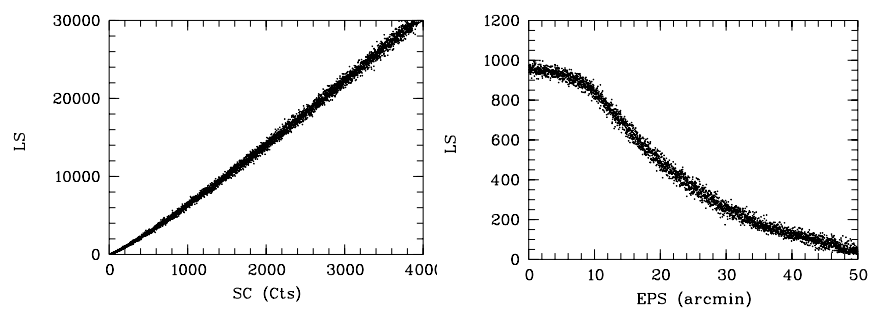

Fig. 5. The source likelihood SP $\hat{L}_{\mathrm{S}}=$ : LS versus the source counts $\Lambda_{\mathrm{S}}=: \mathrm{SC}$ in the range $[10,4000]$ at detector centre $\epsilon_{\mathrm{S}}=0$ (left $)$ and versus the source off-axis angle $\epsilon_{\mathrm{S}}=$ : EPS in the range $\left[0^{\prime}, 50^{\prime}\right]$ for a source of $\Lambda_{\mathrm{S}}=200$ counts with a background density $\lambda_{\mathrm{B}}=10^{-4}$ at PSPC detector amplitudes $A_{\mathrm{B}}=30, A_{\mathrm{S}}=100$ without wobbling.

Let $a, b \in S$ be a pair of distinct source points and $C_{a, b}$ an oriented curve from $a$ to $b$. Let now $s(u), 0 \leq u \leq 1$, be a parameterization of $C_{a, b}$. Then $\hat{e}_{p}(u), \hat{e}_{\sigma_{p}}(u), 0 \leq u \leq 1$ with $\hat{e}_{p}, \hat{e}_{\sigma_{p}}$ from Eq. (34) is a one-parametric stochastic process (SP). We complete the choice of the exploration method by choosing paths being the segments between their endpoints and take all segments parallel to the axes in $S$,

$$
\begin{aligned}
& \mathcal{C}_{\Lambda_{\mathrm{S}}}:=\left\{s \in S: \underline{\Lambda}_{\mathrm{S}} \leq \Lambda_{\mathrm{S}} \leq \bar{\Lambda}_{\mathrm{S}}\right\}, \\
& \mathcal{C}_{\epsilon_{\mathrm{S}}}:=\left\{s \in S: \underline{\epsilon}_{\mathrm{S}} \leq \epsilon_{\mathrm{S}} \leq \bar{\epsilon}_{\mathrm{S}}\right\}, \\
& \mathcal{C}_{v_{\mathrm{S}}}:=\left\{s \in S: \underline{v}_{\mathrm{S}} \leq \nu_{\mathrm{S}} \leq \bar{v}_{\mathrm{S}}\right\}, \\
& \mathcal{C}_{\lambda_{\mathrm{B}}}:=\left\{s \in S: \underline{\lambda}_{\mathrm{B}} \leq \lambda_{\mathrm{B}} \leq \bar{\lambda}_{\mathrm{B}}\right\} .
\end{aligned}
$$

Along the paths from Eq. (36), all but source photon number $\Lambda_{\mathrm{S}}$, source off-axis angle $\epsilon_{\mathrm{S}}$, background count density $\lambda_{\mathrm{B}}$, and source extension parameter $v_{\mathrm{S}}$ remain fixed. In three separate subsections, we will report on the MLE performance with varying source counts $\Lambda_{\mathrm{S}}$, off-axis angle $\epsilon_{\mathrm{S}}$, and varying source extension parameter $v_{\mathrm{S}}$. Only PSPC performance will be shown explicitly. In all simulations to follow a background count density $\lambda_{\mathrm{B}}:=10^{-4}$ counts/sky_pix ${ }^{2}$ was chosen along with a background detector amplitude $A_{\mathrm{B}}=30$ which is softer compared to the source amplitude $A_{\mathrm{S}}=100$. In the case of varying off-axis angle $\epsilon_{\mathrm{S}}$, a Poisson source with $\Lambda_{\mathrm{S}}=200$ counts on average was chosen. A fluctuating source corresponds to the observational reality and the errors for the estimates issued are based on Poisson emitters.

The left part of Fig. 5 shows the likelihood $\hat{L}_{\mathrm{S}}$ of source existence depending on the true source counts $\Lambda_{\mathrm{S}}$ in the range $[10,4000]$. At this scale, the likelihood is almost linearly increasing with a slope of about 5 units per source count. This likelihood, Fig. 5 right, as a function of the source off-axis angle behaves differently. In the most sensitive innermost $10^{\prime}$ PSPC detector region, $\hat{L}_{\mathrm{S}}$ is nearly constant. An almost linear decrease follows up to about $30^{\prime}$. In the remaining outer part the decrease becomes less steep down to likelihoods of about 50 (for a source comprised of 200 mean source counts. This number is based on Table 4).

\subsection{Precision of the source count estimation}

Figure 6 left shows the MLE error SP $\hat{e}_{\Lambda_{S}}:=\hat{\Lambda}_{\mathrm{S}}-\Lambda_{\mathrm{S}}$ of the unvignetted source counts versus the unvignetted source counts $\Lambda_{\mathrm{S}}$ in the range $10=: \underline{\Lambda}_{\mathrm{S}} \leq \Lambda_{\mathrm{S}} \leq \bar{\Lambda}_{\mathrm{S}}:=4000$ counts in steps of
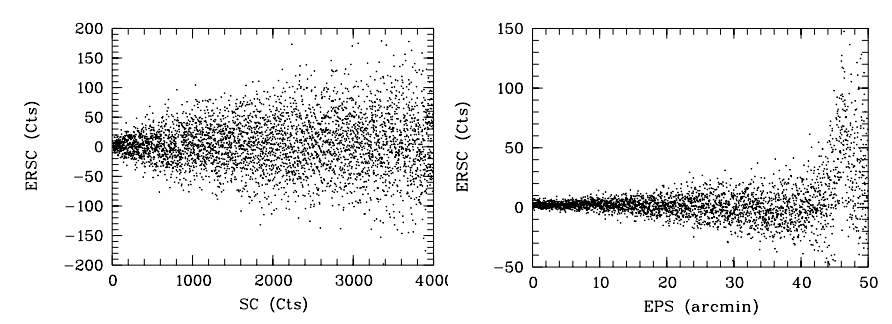

Fig. 6. The unvignetted source count error $\mathrm{SP} \hat{e}_{\Lambda_{\mathrm{S}}}=$ : ERSC versus the source counts $\Lambda_{\mathrm{S}}=: \mathrm{SC}$ in the range $[10,4000]$ at detector centre $\epsilon_{\mathrm{S}}=0^{\prime}$ (left) and versus the source off-axis angle $\epsilon_{\mathrm{S}}=$ : EPS in the range $\left[0^{\prime}, 50^{\prime}\right]$ (right) with a background density $\lambda_{\mathrm{B}}=10^{-4}$ at PSPC detector amplitudes $A_{\mathrm{B}}=30, A_{\mathrm{S}}=100$ without wobbling.
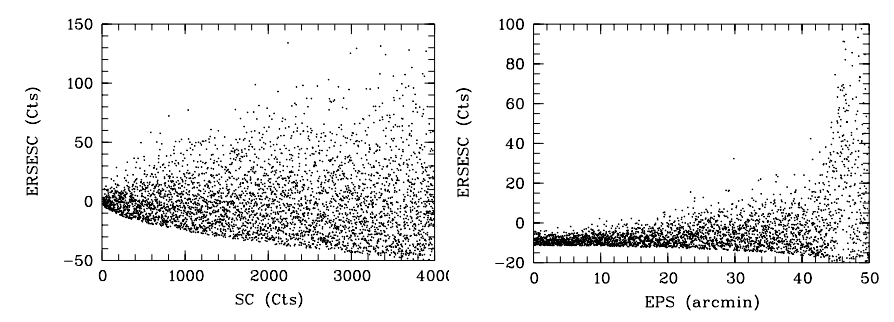

Fig. 7. The error SP of the standard error for the unvignetted source count estimate $\hat{e}_{\sigma_{\Lambda_{\mathrm{S}}}}=$ : ERSESC versus the unvignetted source counts $\Lambda_{\mathrm{S}}=: \mathrm{SC}$ at detector centre $\epsilon_{\mathrm{S}} \approx 0^{\prime}$ (left) and versus the source offaxis angle $\epsilon_{\mathrm{S}}=$ : EPS in the range $\left[0^{\prime}, 50^{\prime}\right]$ (right) with a background density $\lambda_{\mathrm{B}}=10^{-4}$ at a source position $\epsilon_{\mathrm{S}}=0^{\prime}$ and PSPC detector amplitudes $A_{\mathrm{B}}=30, A_{\mathrm{S}}=100$ without wobbling.

one count. The process sample mean taken along $C_{\Lambda_{\mathrm{S}}}$ is $\hat{\mu}_{e_{\Lambda_{\mathrm{S}}}}=$ 4.19 and the standard deviation is $\hat{\sigma}_{e_{\Lambda_{\mathrm{S}}}}=2.34$. We notice a systematic overestimation in the range beginning with about 2.44 counts and ending with about 5.54 . The standard deviation increases moderately with the source strength $\Lambda_{\mathrm{S}}$ from 1.86 to 2.30 cts.

In Fig. 6, right the same error $\hat{e}_{\Lambda_{\mathrm{S}}}$ is shown with its dependence on the off-axis angle $\epsilon_{\mathrm{S}}$. It starts with an unbiased part along with increasing dispersion up to $45^{\prime}$ and beyond shows a sharp rise of the bias due to the large vignetting at the outer detector margin. Note the the large overestimation at around $47^{\prime}$.

Figure 7 left displays the error SP $\hat{e}_{\sigma_{\Lambda_{\mathrm{S}}}}=$ : ERSESC according to Eq. (34) for the unvignetted source counts versus the unvignetted source counts $\Lambda_{\mathrm{S}}=$ : SC. Beginning with an unbiased initial part, a quadratic overestimation of the issued standard error $\hat{\sigma}_{\Lambda_{S}}$ occurs with increasing source strength $\Lambda_{S}$. The standard error has about three times the correct size. The dispersion in Fig. 7 increases slowly with the source strength. The right Fig. 7 shows the standard error SP as a function of the source off-axis angle $\epsilon_{\mathrm{S}}$. We see again a too large standard deviation leading to a negative bias of initially 10 counts. The bias becomes less pronounced with growing off-axis angle and becomes small for about $45^{\prime}$. Beyond this radius the dispersion increases sharply. The non-symmetry of this SP about the mean is noticeable. The lower tail is compressed and the upper one stretched.

The next pair in Fig. 8 shows the error SP for the vignetted source counts $\Lambda_{\mathrm{S}}^{V}$ analogously to the previous unvignetted source counts $\Lambda_{\mathrm{S}}$. Figure 8 left shows the MLE error 

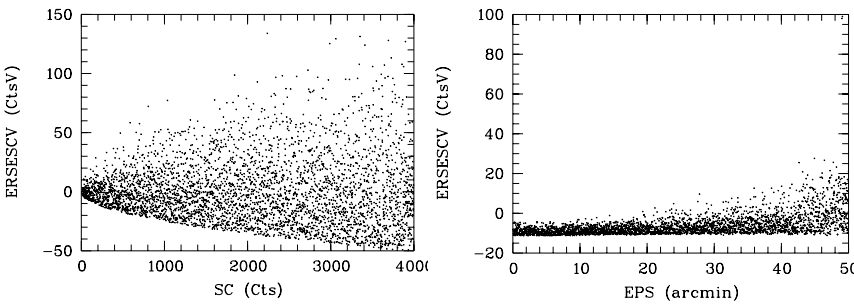

Fig. 8. The vignetted source count error SP $\hat{e}_{\Lambda_{\mathrm{S}}}^{V}=$ : ERSCV versus the unvignetted source counts $\Lambda_{\mathrm{S}}=: \mathrm{SC}$ for a source position $\epsilon_{\mathrm{S}} \approx 0^{\prime}$ (left) and versus the source off-axis angle $\epsilon_{\mathrm{S}}=$ : EPS for $\Lambda_{\mathrm{S}}:=200$ source counts (right) with a background density $\lambda_{\mathrm{B}}=10^{-4}$ and with a background density $\lambda_{\mathrm{B}}=10^{-4}$ at PSPC detector amplitudes $A_{\mathrm{B}}=30, A_{\mathrm{S}}=$ 100 without wobbling.
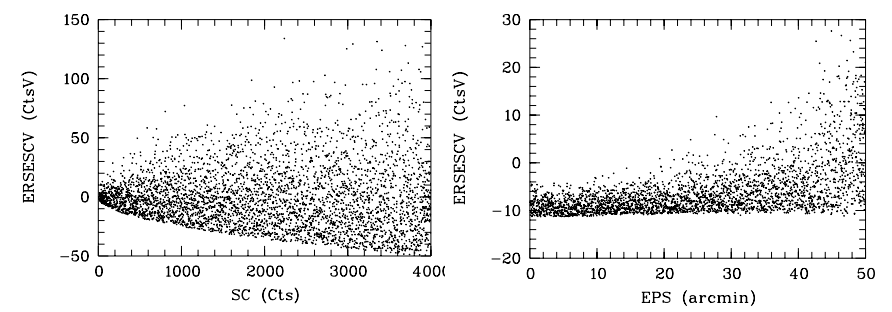

Fig. 9. The error SP for standard error $\hat{e}_{\sigma_{\Lambda} V}=$ : ERSESCV versus the source $\Lambda_{\mathrm{S}}=: \mathrm{SC}$ in the range $[10,4000]$ at detector centre $\epsilon_{\mathrm{S}} \approx 0^{\prime}$ (left) and versus the source off-axis angle $\epsilon_{\mathrm{S}}=:$ EPS in the range $\left[0^{\prime}, 50^{\prime}\right]$ for $\Lambda_{\mathrm{S}}:=200$ source counts (right) with a background density $\lambda_{\mathrm{B}}=10^{-4}$ at the PSPC detector amplitudes $A_{\mathrm{B}}=30, A_{\mathrm{S}}=100$ without wobbling.

SP $\hat{e}_{\Lambda_{\mathrm{S}}^{V}}:=\hat{\Lambda}_{\mathrm{S}}^{V}-\Lambda_{\mathrm{S}}^{V}$ versus the unvignetted source counts $\Lambda_{\mathrm{S}}$ in the range $[10,4000]$ counts in steps of one count. We see a bias of $\hat{\mu}_{\Lambda_{s}^{v}}=2.97$ counts at a dispersion $\hat{\sigma}_{\Lambda_{s}^{v}}=2.21$ counts. Both mean and dispersion are almost constant within the whole range. In the right Fig. 8, the same error SP $\hat{e}_{\Lambda_{\mathrm{S}}^{v}}$ is shown as a function of the source off-axis angle $\epsilon_{\mathrm{S}}$. Here mean and standard error are $\hat{\mu}_{\Lambda_{\mathrm{s}}^{v}}=1.81 \mathrm{cts}$ and $\hat{\sigma}_{\Lambda_{\mathrm{S}}^{v}}=7.39 \mathrm{cts}$. The latter increases with increasing $\epsilon_{\mathrm{S}}$ from 7.39 to 16.24 counts. The mean is almost constant up to $45^{\prime}$ and decreases beyond to $\hat{\mu}_{\Lambda_{\mathrm{S}}^{V}}=-4.15$ counts.

The SP for the standard error $\hat{\sigma}_{\Lambda_{\mathrm{S}}^{V}}$ is shown in Fig. 9. We observe a quadratic overestimation with increasing source strength. Finally, the dependence of the same standard error SP $\hat{e}_{\sigma_{\Lambda}^{V}}$ on the source off-axis angle $\epsilon_{\mathrm{S}}$ is shown. We see a decreasing overestimation of the error of about $\approx 13$ counts which becomes less pronounced with increasing source off-axis angle $\epsilon_{\mathrm{S}}$. Thus, for weak sources, the ROSAT $1 \sigma$-standard error is too conservative and becomes more realistic for stronger sources.

\subsection{Precision of the positional estimation}

Figure 10 left shows the SP of the first positional errors $\hat{e}_{X_{\mathrm{S}}}:=$ $\hat{X}_{\mathrm{S}}-X_{\mathrm{S}}$ of the ML estimate $\hat{X}_{\mathrm{S}}$ as a function of the source photon count number $\Lambda_{S} \in[10,4000]$. Evidently, the distribution spread $\hat{\sigma}_{e_{X_{\mathrm{S}}}}\left(\Lambda_{\mathrm{S}}\right)$ becomes smaller: 1.3 sky_pix near the left end of the range, 0.47 sky_pix in the middle range, and 0.33 sky_pix right in Fig. 10, i.e. for increasing source photon numbers $\Lambda_{\mathrm{S}}$. This is consistent with the expected $O\left(1 / \sqrt{\Lambda_{\mathrm{S}}^{V}}\right)$ behaviour for large numbers of photons $\Lambda_{\mathrm{S}}^{V} \rightarrow \infty$. No bias
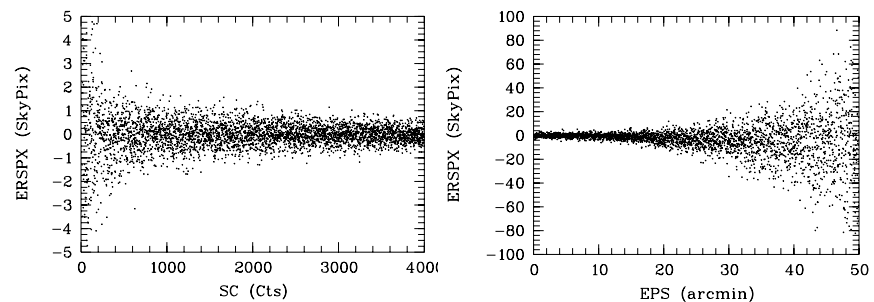

Fig. 10. The first positional error SP $\hat{e}_{X_{S}}=$ : ERSPX in units of sky pixels $=0.5^{\prime \prime}$ versus the source photons $\Lambda_{S}$ in the range $[10,4000]$ at detector centre $\epsilon_{\mathrm{S}}=0^{\prime}$ (left) and versus the source off-axis angle $\epsilon_{\mathrm{S}}=$ : EPS in the range $\left[0^{\prime}, 50^{\prime}\right] \Lambda_{\mathrm{S}}:=200$ source counts (right) with a background density $\lambda_{\mathrm{B}}=10^{-4}$ at the PSPC detector amplitudes $A_{\mathrm{B}}=$ $30, A_{\mathrm{S}}=100$ without wobbling.
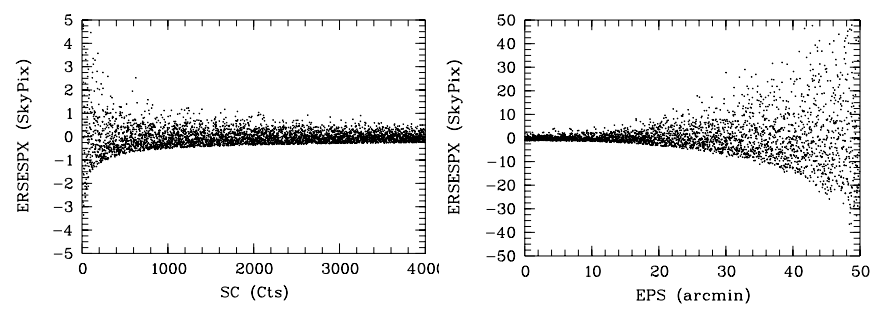

Fig. 11. The error SP for the first standard error $\hat{e}_{\sigma_{X_{\mathrm{S}}}}=$ : ERSESPX in units of sky pixels $=0.5^{\prime \prime}$ to the source counts $\Lambda_{\mathrm{S}}=$ : SC in the range $[10,4000]$ at detector centre $\epsilon_{\mathrm{S}}=0^{\prime}$ (left) and versus the source offaxis angle $\epsilon_{\mathrm{S}}=$ : EPS in the range $\left[0^{\prime}, 50^{\prime}\right]$ (right) with a background density $\lambda_{\mathrm{B}}=10^{-4}$ at the PSPC detector amplitudes $A_{\mathrm{B}}=30, A_{\mathrm{S}}=100$ without wobbling.

around the first axis $\hat{e}_{X_{\mathrm{S}}}=0$ is present in the entire range of $\Lambda_{\mathrm{S}}$. The sample mean is $\hat{\mu}_{e_{X_{\mathrm{S}}}}=0.017$ sky_pix and stays uniform at a few hundredths of a sky_pix within the whole range. The behaviour with increasing source off-axis angle $\epsilon_{\mathrm{S}}$ is shown in Fig. 10, right. The estimate $\hat{X}_{\mathrm{S}}$ is unbiased at $\epsilon_{\mathrm{S}}=0^{\prime}$ and stays nearly unbiased until $\epsilon_{\mathrm{S}}=50^{\prime}$. The increase of the spread $\hat{\sigma}_{e_{X_{\mathrm{S}}}}$ of the distribution with increasing $\epsilon_{\mathrm{S}}$ is larger. It varies from 1.53 sky_pix for small $\epsilon_{\mathrm{S}}$ to $9.50 \mathrm{sky} \_$pix in the middle to 152.53 sky_pix at the upper end of the range.

Figure 11 shows the positional standard errors in the first direction. The sample mean $\hat{\mu}_{e_{\mathrm{S}}}=-0.016 \mathrm{sky} \_$pix means that no bias is present. The sample standard deviation $\hat{\sigma}_{e_{X_{S}}}$ decreases from 1.018 over 0.28 to 0.19 sky_pix at the upper end of the range. The lower parts in Fig. 11 are denser than the upper tails. This means an asymmetry of the sample distribution about the mean.

A similar behaviour is expected and observed for the second positional error $\hat{e}_{Y_{\mathrm{S}}}:=\hat{Y}_{\mathrm{S}}-Y_{\mathrm{S}}$ in Fig. 12. We see again unbiased estimates along with a spread decreasing with the source counts $\Lambda_{\mathrm{S}}$ and increasing with the off-axis angle $\epsilon_{\mathrm{S}}$ all with the same range as for the first direction $X_{\mathrm{S}}$ in 10 .

\subsection{Precision of the source extent estimation}

This section reports the non-extended sources, $v_{\mathrm{S}}=0$, then the extended sources, $v_{\mathrm{S}}>0$.

With the ML estimate $\hat{v}_{S}$ we form according to Eq. (34) the error SP $\hat{e}_{v_{\mathrm{S}}}:=\hat{v}_{\mathrm{S}}-v_{\mathrm{S}}$. In Fig. 14, this error SP is plotted against the source strength $\Lambda_{S} \in[10,4000]$ (left) and versus the source 

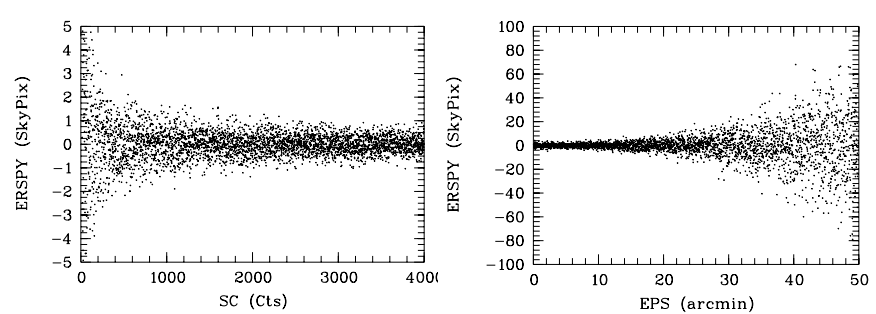

Fig. 12. Second positional error SP $\hat{e}_{Y_{\mathrm{S}}}=$ : ERSPY in units of sky pixels $=0.5^{\prime \prime}$ versus the source photons $\Lambda_{S}=: S C$ in the range $[10,4000]$ at detector centre $\epsilon_{\mathrm{S}}=0^{\prime}$ (left) and versus the source off-axis angle $\epsilon_{\mathrm{S}}=$ : EPS in the range $\left[0^{\prime}, 50^{\prime}\right]$ (right) with a background density $\lambda_{\mathrm{B}}=10^{-4}$ at the PSPC detector amplitudes $A_{\mathrm{B}}=30, A_{\mathrm{S}}=100$ without wobbling.
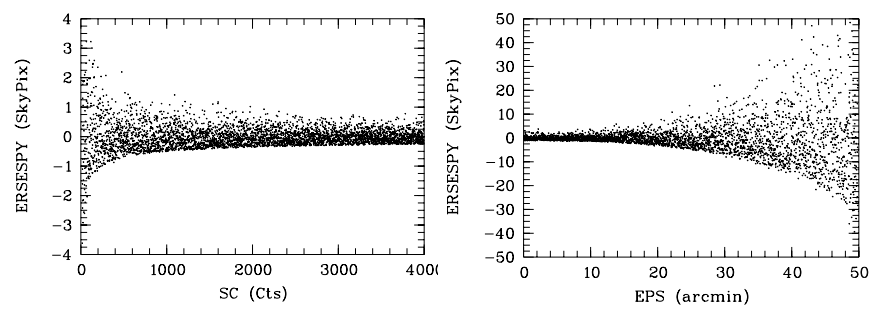

Fig. 13. The error SP for the second positional error $\hat{e}_{Y_{\mathrm{S}}}=$ : ERSESPY in units of sky pixels $=0.5^{\prime \prime}$ versus the source photons counts $\Lambda_{\mathrm{S}}=$ : $\mathrm{SC}$ in the range $[0,4000]$ at detector centre $\epsilon_{\mathrm{S}}=0^{\prime}$ (left) and versus the source off-axis angle $\epsilon_{\mathrm{S}}=$ : EPS in the range $\left[0^{\prime}, 50^{\prime}\right]$ (right) with a background density $\lambda_{\mathrm{B}}=10^{-4}$ at the PSPC detector amplitudes $A_{\mathrm{B}}=$ $30, A_{\mathrm{S}}=100$ without wobbling.
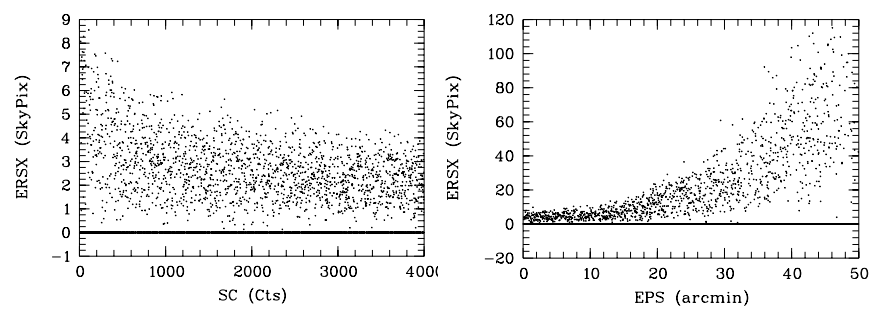

Fig. 14. The source extent error SP $\hat{e}_{v_{\mathrm{S}}}=$ : ERSX versus the source strength $\Lambda_{\mathrm{S}}=$ : SC in the range $[10,4000]$ at detector centre $\epsilon_{\mathrm{S}}=0^{\prime}$ (left) and versus the source off-axis angle $\epsilon_{\mathrm{S}}=$ : EPS in the range $\left[0^{\prime}, 50^{\prime}\right]$ (right) with a background density $\lambda_{\mathrm{B}}=10^{-4}$ at the PSPC detector amplitudes $A_{\mathrm{B}}=30, A_{\mathrm{S}}=100$ without wobbling.

off-axis angle $\epsilon_{\mathrm{S}}$ in the range $\left[0^{\prime}, 50^{\prime}\right]$. The cases in which the true source extent and the estimated extent coincide, $\hat{v}_{\mathrm{S}}=v_{\mathrm{S}}$, lie on the horizontal line $\hat{e}_{v_{\mathrm{S}}}=0$.

In Fig. 15, the error SP for the standard deviation $\hat{e}_{\sigma_{v_{\mathrm{S}}}}$ is shown. The upper envelope stems from the error-free estimation cases. Indispensable for the assessment of Fig. 15 are the related likelihoods for the presence of an extended source $\hat{L}_{\mathrm{SE}}:=L\left(\hat{v}_{\mathrm{S}}\right)-L(0)$ in Fig. 16, i.e. for $v_{\mathrm{S}}>0$. Under an applied threshold for the source extent likelihood $L_{\mathrm{SE}}^{0}=8$, no source was found to be extended.

We now turn to the estimation of positive ML source extents $v_{\mathrm{S}}>0$. Fig. 17 displays the likelihood of source extent $\hat{L}_{\mathrm{SE}}$ versus the source extent parameter $v_{\mathrm{S}}$ in the range $[0,120]$ sky_pix. The count quotient $\Lambda_{S} / \Lambda_{B}$ was kept constant with respect to $v_{\mathrm{S}}$. After a linear increase up to a source extension of about 50 sky_pix, the likelihood of source extension
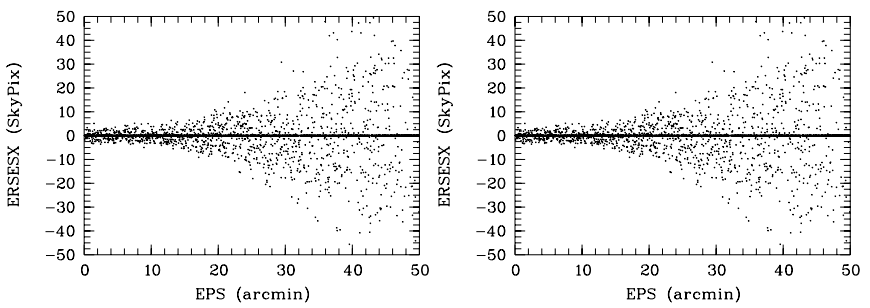

Fig. 15. The error SP for the standard error $\hat{e}_{\sigma_{v_{\mathrm{S}}}}\left(\Lambda_{\mathrm{S}}\right)=$ : ERSESX for the source extent $v_{\mathrm{S}}$ versus the source strength $\Lambda_{\mathrm{S}}=: \mathrm{SC}$ in the range $[10,4000]$ at detector centre $\epsilon_{\mathrm{S}}=0^{\prime}$ (left) and versus the source strength $\Lambda_{\mathrm{S}}=$ : SC in the range $[10,4000]$ (right) with a background density $\lambda_{\mathrm{B}}=10^{-4}$ at the PSPC detector amplitudes $A_{\mathrm{B}}=30, A_{\mathrm{S}}=100$ without wobbling.
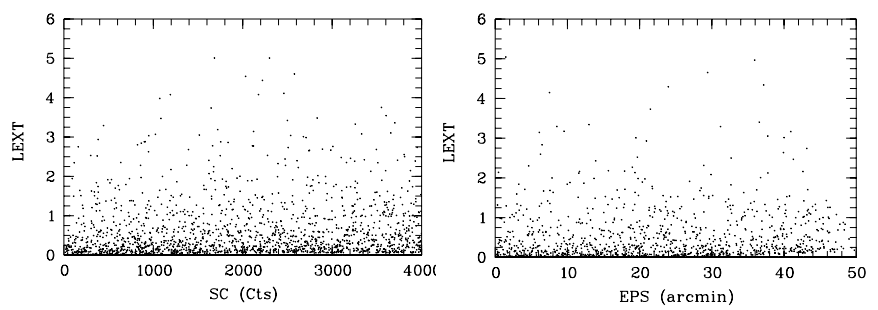

Fig. 16. The likelihood of the source extent $\hat{L}_{v_{\mathrm{S}}}\left(\Lambda_{\mathrm{S}}\right)=$ : LEXT versus the source counts $\Lambda_{\mathrm{S}}=$ : SC in the range $[10,4000]$ at detector centre $\epsilon_{\mathrm{S}}=0^{\prime}($ left $)$ and versus the source off-axis angle $\epsilon_{\mathrm{S}}=$ : EPS in the range $\left[0^{\prime}, 50^{\prime}\right](r i g h t)$ with a background density $\lambda_{\mathrm{B}}=10^{-4}$ at the PSPC detector amplitudes $A_{\mathrm{B}}=30, A_{\mathrm{S}}=100$ without wobbling.
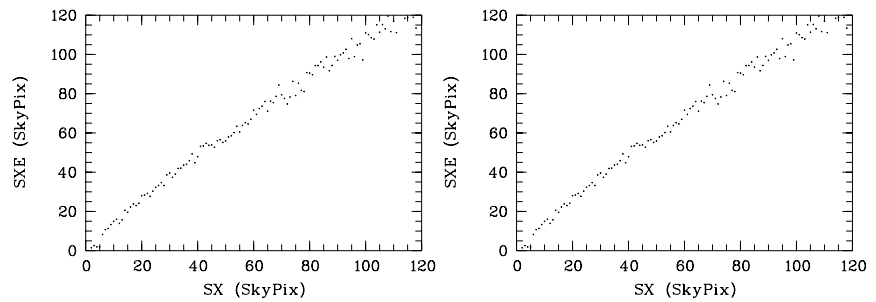

Fig. 17. The likelihood of the source extent $\hat{L}_{v_{\mathrm{S}}}=$ : LEXT versus the source extent $v_{\mathrm{S}}=: \mathrm{SX}$ in the range $[0,120]$ sky_pix at detector centre $\epsilon_{\mathrm{S}}=0^{\prime}($ left $)$ and the estimated source extent $\hat{v}_{\mathrm{S}}=:$ SXE in the range $[0,120]$ sky_pix (right) with a background density $\lambda_{\mathrm{B}}=10^{-4}$ (for $v_{\mathrm{S}}=0$ ) at the PSPC detector amplitudes $A_{\mathrm{B}}=30, A_{\mathrm{S}}=100$ without wobbling and $\Lambda_{\mathrm{S}}=200$ source counts.

$\hat{L}_{\mathrm{SE}}$ increases sub-linearly and becomes also more scattered. The right picture shows the source extension estimate $\hat{v}_{\mathrm{S}}$. One observes a developing moderate overestimation with increasing source extension. For $v_{\mathrm{S}}>40$ sky_pix, the overestimation amounts to $\hat{\mu}_{v_{\mathrm{S}}>40}=8.03$ sky_pix and with standard deviation $\hat{\sigma}_{v_{\mathrm{S}}>40}=3.6$ sky_pix.

The standard error estimate $\hat{\sigma}_{v_{\mathrm{S}}}$ and its error SP $\hat{e}_{\sigma_{v_{\mathrm{S}}}}$ are presented next in Fig. 18. The standard error is correct at low $v_{\mathrm{S}}$ and develops, beyond $v_{\mathrm{S}}=40$ sky_pix, a linearly progressing underestimation with increasing source extension of about 0.25 sky_pix per sky_pix. More quantitatively, $\hat{\mu}_{e_{\sigma_{v_{\mathrm{S}}} \leq 40}}=$ 8.03 sky_pix was deemed correct.

Too small source cut radii $r_{\mathrm{S}}:=\rho_{\mathrm{S}}$ FWHM result in a noticeable loss in source detection likelihood $\hat{L}_{\mathrm{SE}}$ as well as in source photon counts $\hat{\Lambda}_{\mathrm{S}}$. In the foregoing simulations this 

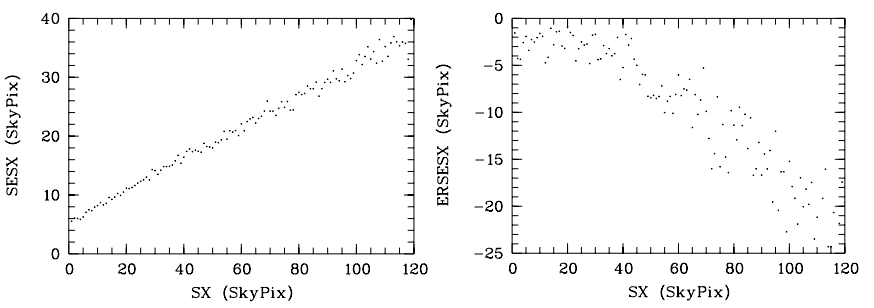

Fig. 18. The standard error $\hat{e}_{v_{\mathrm{S}}}=$ : SESX for the source extent estimate $\hat{v}_{\mathrm{S}}=: \mathrm{SX}$ versus the source extent $\Lambda_{\mathrm{S}}=: \mathrm{SX}$ in the range $[0,120]$ sky_pix at detector centre $\epsilon_{\mathrm{S}}=0^{\prime}$ (left) and versus the source off-axis angle $\epsilon_{\mathrm{S}}=$ : EPS in the range $\left[0^{\prime}, 50^{\prime}\right]$ (right) with a background density $\lambda_{\mathrm{B}}=10^{-4}$ (for $v_{\mathrm{S}}=0$ ) at the PSPC detector amplitudes $A_{\mathrm{B}}=30, A_{\mathrm{S}}=100$ without wobbling and $\Lambda_{\mathrm{S}}=200$ source counts.

radius was therefore set to $\rho_{\mathrm{S}}:=10$. In the standard setting, $\rho_{\mathrm{S}}=2.5$, a censoring effect is in effect from the middle of the above range $[0,120]$ onwards. In earlier EXSAS versions, the likelihood of source extension $\hat{L}_{\mathrm{SE}}$ was underestimated for $v_{\mathrm{S}}>0$.

\subsection{Confusing groups of sources}

Consider a pair of point sources or, more generally, a group of point sources within a distance $d$ sky_pix from a common center. Now, express appropriately the distance from the center in units of the PSF radius, $d=: \delta \cdot r_{\mathrm{PSF}}$. Start with $\delta=2$, say, and make the distance $\delta$ smaller and smaller to $\delta \ll 1$ to obtain a group of confluent sources. The behaviour of the MLE performance in this process is clear: with decreasing distance $\delta$, the error bars become larger. A single-source detection method must eventually fail. To lower the radius $\delta_{\text {fail }}$ of failure, a source estimation procedure for source groups was established in EXSAS and mentioned in Paper II. The quantitative aspects of the deteriorating quality of the source parameters and the related error bars will not be studied in this work.

\subsection{Summary of the simulation results in pointing mode}

The next table summarizes in the $P(\mu, \sigma)$ column the errors with respect to source counts, position, and extension (Extens.) for the PSPC in terms of the means $\mu$ and standard deviations $\sigma$ from the foregoing Sects. 4.3 to 4.5 in the four most respresentative source strength classes: 10 to 24,25 to 49,50 to 99, and 100 to 199 source counts. The analogous results for the HRI detector obtained in the same way as described before are supplemented in the column $H(\mu, \sigma)$. For each detector and each source class 5000 sources (uniformly distributed within the source classes) in on-axis position were simulated and subjected to analysis. A representative positional uniform background count rate of $0.56 \mathrm{cts} \cdot\left({ }^{\prime}\right)^{-2}$ was calculated from the means for exposure times and background count rates from the BSC column in Table 4. For the PSPC (with 256 channels), the background detector amplitudes fell into the channel $A_{\mathrm{B}}=30$; the source photons were harder, $A_{\mathrm{S}}=100$. In the case of the HRI (possessing 8 channels) detector the channel
Table 3. The ROSAT MLE source parameter precisions summary ( $\mu=$ mean, $\sigma=$ standard deviation) for the ROSAT detectors PSPC (P) and HRI $(\mathrm{H})$ in pointing mode obtained by simulated observations in four observed source count (SourceCnt) ranges 10-24 to 100-199 in on-axis source position and with $0.56 \mathrm{cts} /\left({ }^{\prime}\right)^{2}$ background count rate. Entry 0.1 means: $\leq 0.1$.

\begin{tabular}{lrrl}
\hline \hline Source Param. & $P(\mu, \sigma)$ & $H(\mu, \sigma)$ & Source Cnt. \\
\hline Srce counts/[cts] & $(-0.7,4.2)$ & $(-1.2,4.1)$ & $10-2$ \\
Srce position/["] & $(3.6,91)$ & $(0.8,5.7)$ & $10-24$ \\
Srce extens./["] & $(-0.3,1.4)$ & $(-0.1,0.6)$ & $10-24$ \\
\hline Srce counts/[cts] & $(-1.9,5.7)$ & $(-0.1,0.9)$ & $25-4$ \\
Srce position/["]] & $(0.1,6.4)$ & $(0.1,0.6)$ & $25-49$ \\
Srce Extens./["] & $(-0.1,1.6)$ & $(-0.1,0.4)$ & $25-49$ \\
\hline Srce counts/[cts] & $(-4.1,8.4)$ & $(-4.8,8.3)$ & $50-99$ \\
Srce position/["] & $(0.1,3.4)$ & $(0.1,0.4)$ & $50-99$ \\
Srce Extens./["] & $(0.1,1.7)$ & $(0.1,0.6)$ & $50-99$ \\
\hline Srce counts/[cts] & $(-8.8,11.7)$ & $(-9.6,11.6)$ & $100-199$ \\
Srce position/[["] & $(0.1,0.8)$ & $(0.1,0.3)$ & $100-199$ \\
Srce Extens./["] & $(0.1,1.9)$ & $(0.1,0.3)$ & $100-199$ \\
\hline
\end{tabular}

numbers were $A_{\mathrm{B}}=2$ and $A_{\mathrm{S}}=8$. The standard setting $\rho_{\mathrm{S}}=2.5$ for the cut radius factor was applied. (Consult Fig. 17 for PSPC sources in larger off-axis position.)

The HRI detector possesses a higher positional resolution than the PSPC detector. Compared with the PSPC, the positional HRI standard deviations are smaller. However, conceptually, detector resolution and accuracy for a measured quantity are independent characteristics. Table 3 shows us also that the detector resolution is not the limiting factor for the achieved positional accuracy.

\section{Catalogue comparisons}

We can perform a quality assessment of the ROSAT source detection procedure by catalogue comparisons.

Assume a pair B, C of catalogues has a nonvoid subset $\mathcal{S}_{\mathrm{B}, \mathrm{C}}$ of $M$ common sources. Denote by $\mathrm{C}$ the reference catalogue from which the other, B, is evaluated. Let both catalogues, possibly based on observations in different wavelength regimes, contain at least source directions and possibly also source fluxes. Let the positions be referred to the equatorial system of the same epoch with right ascension $\alpha \in\left[0^{\circ}, 360^{\circ}\right)$ and declination $\delta \in\left[-90^{\circ},+90^{\circ}\right]$. Assume further that standard errors for specified confidence levels $l^{\mathrm{B}}, l^{\mathrm{C}} \in(0,1)$ are given for the source flux $\lambda$ and source position $(\alpha, \delta)$. From the source entries $s^{\mathrm{B}} \in \mathrm{B}, s^{\mathrm{C}} \in \mathrm{C}$, we form the intra-catalogue source deviation entry $\Delta s^{\mathrm{B}-\mathrm{C}}$,

$$
\begin{aligned}
\Delta s^{\mathrm{B}-\mathrm{C}} & :=\left(s^{\mathrm{B}-\mathrm{C}}, \sigma^{\mathrm{B}-\mathrm{C}}\right), \\
s^{\mathrm{B}-\mathrm{C}} & :=\left(\lambda^{\mathrm{B}}-\lambda^{\mathrm{C}}, \alpha^{\mathrm{B}}-\alpha^{\mathrm{C}}, \delta^{\mathrm{B}}-\delta^{\mathrm{C}}\right), \\
\sigma^{\mathrm{B}-\mathrm{C}} & :=\left(\sigma_{\lambda}^{\mathrm{B}}+\sigma_{\lambda}^{\mathrm{C}}, \sigma_{\alpha}^{\mathrm{B}}+\sigma_{\alpha}^{\mathrm{C}}, \sigma_{\delta}^{\mathrm{B}}+\sigma_{\delta}^{\mathrm{C}}\right), \\
s^{\mathrm{A}} & :=\left(\lambda^{\mathrm{A}}, \alpha^{\mathrm{A}}, \delta^{\mathrm{A}}, \sigma_{\lambda}^{\mathrm{A}}, \sigma_{\alpha}^{\mathrm{A}}, \sigma_{\delta}^{\mathrm{A}}\right), \mathrm{A} \in\{\mathrm{B}, \mathrm{C}\} .
\end{aligned}
$$

The addition law for error bars in Eq. (37) leads to larger sum error bars than those resulting under a normality assumption. 
From Eq. (37) we derive the distance $d_{\xi}^{\mathrm{B}-\mathrm{C}} \geq 0$ between the error bars for the source parameter $\xi \in\{\lambda, \alpha, \delta\}$ and the Boolean specification function $d^{\mathrm{B}-\mathrm{C}} \in\{0,1\}$ by

$d_{\xi}^{\mathrm{B}-\mathrm{C}}\left(s^{\mathrm{B}}, s^{\mathrm{C}}\right):=\max \left\{0,\left|\xi^{\mathrm{B}}-\xi^{\mathrm{C}}\right|-\sigma_{\xi}^{\mathrm{B}}-\sigma_{\xi}^{\mathrm{C}}\right\}$,

$d^{\mathrm{B}-\mathrm{C}}\left(s^{\mathrm{B}}, s^{\mathrm{C}}\right):=\operatorname{sgn}\left(\max \left\{d_{\lambda}^{\mathrm{B}-\mathrm{C}}, d_{\alpha}^{\mathrm{B}-\mathrm{C}}, d_{\delta}^{\mathrm{B}-\mathrm{C}}\right\}\right)$.

By the foregoing definition (38), $d_{\xi}^{\mathrm{B}-\mathrm{C}}=0$ means that the error bars for $\xi$ overlap. Thus, in this case the source is within the specifications for $\xi$ on the significance levels $l_{\xi}^{\mathrm{B}}, l_{\xi}^{\mathrm{C}}$ in both catalogues. Separating error bars, $d_{\xi}^{\mathrm{B}-\mathrm{C}}>0$, must occur in the correct small frequency. On average, this is the fraction $1-\left(l_{\xi}^{\mathrm{B}}+l_{\xi}^{\mathrm{C}}\right) / 2$ of all cases. For $d^{\mathrm{B}-\mathrm{C}}=0$, the twofold catalogued source $\left(s^{\mathrm{B}}, s^{\mathrm{C}}\right)$ under consideration is within specifications for all three source parameters. For $d^{\mathrm{B}-\mathrm{C}}=1$ this is not the case. Also the latter case must occur with the (much) smaller probability

$$
\begin{aligned}
p\left(s^{\mathrm{R}}, s^{\mathrm{T}}\right):= & {\left[1-\left(l_{\lambda}^{\mathrm{B}}+l_{\lambda}^{\mathrm{C}}\right) / 2\right] \cdot\left[1-\left(l_{\alpha}^{\mathrm{B}}+l_{\alpha}^{\mathrm{C}}\right) / 2\right] . } \\
& {\left[1-\left(l_{\delta}^{\mathrm{B}}+l_{\delta}^{\mathrm{C}}\right) / 2\right] . }
\end{aligned}
$$

If the out-of-specification cases occur more/less frequently, the error bars are too small/large and are wrong.

In this work, one of the catalogues $B, C$ must be necessarily a ROSAT catalogue, $B:=R$. An appropriate choice for the reference catalogue is $C:=T$,

$$
\begin{aligned}
\mathrm{R} & :=\text { ROSAT All-Sky Bright Source Catalogue; } \\
\mathrm{T} & :=\mathrm{TYCHO} \text { star catalogue; } \\
\mathrm{M} & :=6456 .
\end{aligned}
$$

The ROSAT catalogue R was presented by Voges et al. (1999), and by Høg et al. (1998) for the TYCHO catalogue. The latter contains optical star positions which are far more precise than the $R$ positions. Our sample size 6456 (based on a search radius of $120^{\prime \prime}$ ) does not allow the consideration of the distribution with respect to right ascension and declination. Hence, the result is averaged over all common observation directions. The consideration of the errors separately for both coordinates is also not carried out. Instead, one quantity, the positional distance will be investigated. The basic assumption is that the $\left(s^{\mathrm{R}}, s^{\mathrm{T}}\right)$ pairing is correct. This is shown to be untrue in a low percentage in Fig. 8 of Voges et al. (1999). A correction for the chance coincidences, however, was deliberately not carried out. So, they remain visible (less than $4 \%$ within $40^{\prime \prime}$ ) in the following histograms.

The next two figures (Figs. 19, 20) show sample distributions with respect to the angular positional distance $d_{\alpha, \delta}^{R-T}$ between the $R$ source relative to the $T$ source counterpart. Comparing the positional precision in $R$ and $T$, it is justified to consider $T$ error-free.

To investigate also the quality of the $R$ error assignment, the whole source population is split into two subpopulations. The first is the one comprising all sources for which the positional distance remains within the $R$ error specification. The second comprises the others, i.e. those which are not within the error specification. The theoretical mutual proportions are determined by the significance level $l$ for the $R$ errors. The source

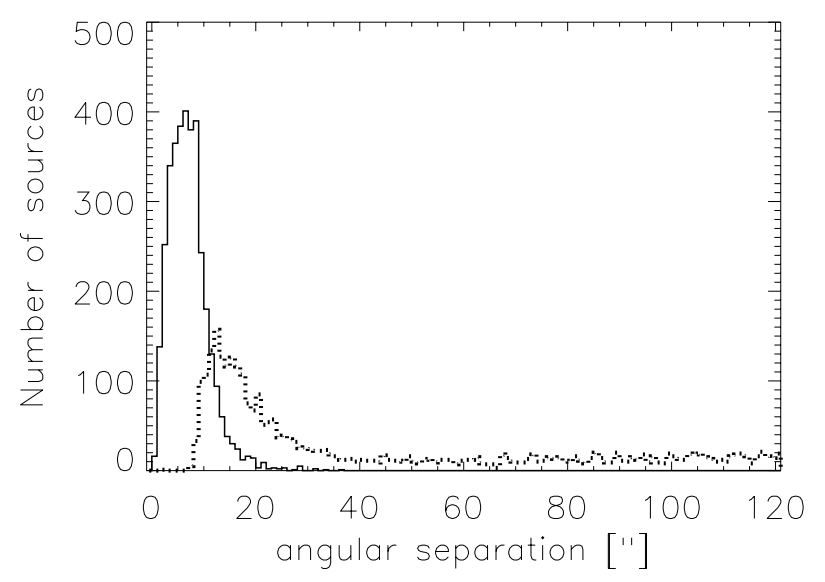

Fig. 19. The source frequency histogram w.r.t. angular source separation $d_{\alpha, \delta}^{R-T}$. Solid subpopulation $=$ within ROSAT error specification; dotted $=$ not within ROSAT error specification.

frequency in the $k$ th histogram bin out of $K=120$ bins for subpopulation $j$ is denoted by $n_{j, k}^{R-T}$. The bin width of $\Delta d:=1^{\prime \prime}$ amounts to the distance domain of 2 arcmin,

$n_{1, k}^{R-T}:=\sum 1\left[k \Delta d \leq d_{\alpha, \delta}^{R-T}<(k+1) \Delta d, d_{\alpha, \delta}^{R-T} \leq \sigma_{\alpha, \delta}^{R-T}\right]$,

$n_{2, k}^{R-T}:=\sum 1\left[k \Delta d \leq d_{\alpha, \delta}^{R-T}<(k+1) \Delta d, d_{\alpha, \delta}^{R-T}>\sigma_{\alpha, \delta}^{R-T}\right]$,

$d_{\alpha, \delta}^{R-T}:=\sqrt{\left(\alpha^{R}-\alpha^{T}\right)^{2}+\left(\delta^{R}-\delta^{T}\right)^{2}}$,

$\sigma_{\alpha, \delta}^{R-T}:=\sqrt{\left(\sigma_{\alpha}^{R}\right)^{2}+\left(\sigma_{\delta}^{R}\right)^{2}}$.

In Eq. (41) the Boolean function $1[E]:=1$ if the expression $E$ is true, otherwise $1[E]:=0$ is used. The sums run over the common sample $\mathcal{S}_{R, T}$ of both catalogues.

The histogram in Fig. 19 shows the sample frequencies for the two subpopulations with a total of 6456 sources. The dotted histogram belongs to the out-of-specification subpopulation. Its distribution tail contains the chance coincidences. Evidently, it is smaller, less narrow and shifted to greater distances. The mean separations in both subpopulations are about 7 " and, when neglecting the tail in the dotted population, $13^{\prime \prime}$. The standard deviation in the first is $4^{\prime \prime}$ (rounded).

The normalized cumulative counterpart to Fig. 19 allows a quantitative assessment of the development of the source fractions with increasing separation distance and across both subpopulations. It contains in the $k$ th bin in subpopulation $j$ the fraction $N_{j, k}^{R-T}$ of the total number of sources,

$N_{j, k}^{R-T}:=\frac{\left(n_{j, 0}+n_{j, 1}+\cdots+n_{j, k}\right)}{N_{1, K}^{R-T}+N_{2, K}^{R-T}}, \quad j=1,2$.

Figure 20 shows the normalized cumulative counterpart histogram. For our $1 \sigma R$ errors, the theoretically expected proportions are $N_{1, K}=68.4 \%$ and $N_{2, K}=31.6 \%$. The observed corresponding fractions are $\hat{N}_{1, K}=3539 / 6456=54.82 \%$ and $\hat{N}_{2, K}=2917 / 6456=45.18 \%$. The chance coincidences are seen in the tail slope in the dotted histogram. The deviation of about $15 \%$ from the theoretical values is not too surprising with regard to the unmodelled error sources.

The sample distribution histogram with respect to the positional error $\sigma_{\alpha, \delta}^{R-T}$ in the two source subpopulations is depicted 


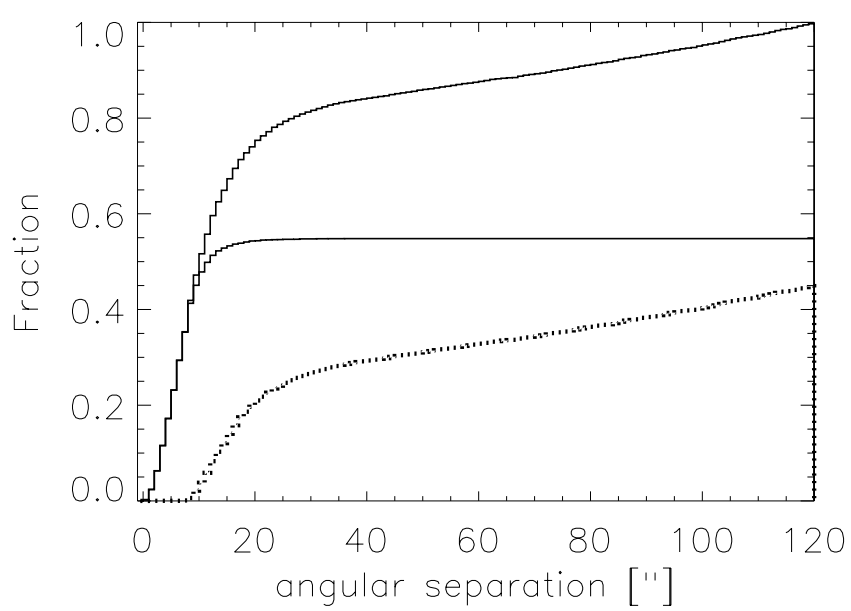

Fig. 20. The normalized cumulative sample source distribution w.r.t. angular source separation $\delta_{\alpha, \delta}^{R-T}$. Large solid population = total source population; solid subpopulation $=$ within ROSAT error specification; dotted $=$ not within ROSAT error specification.

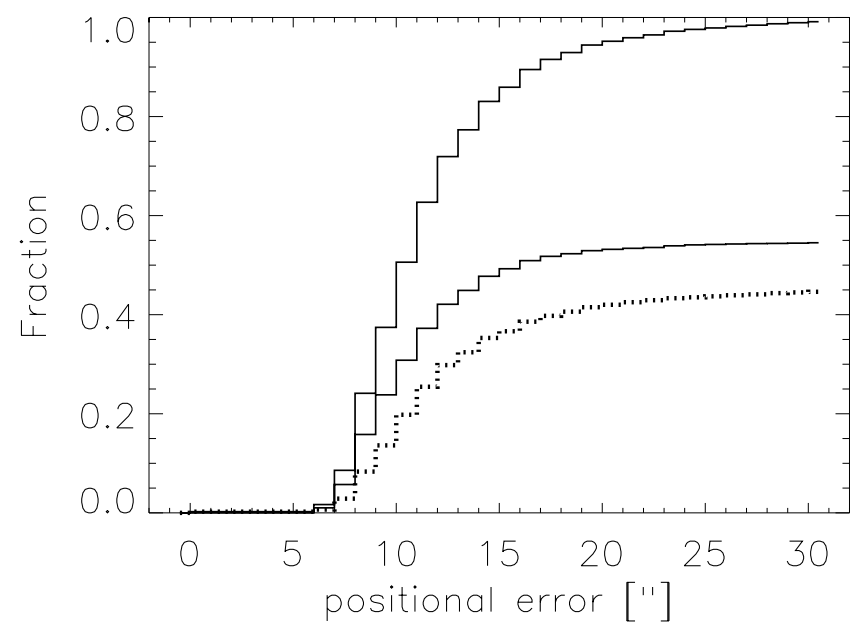

Fig. 21. The cumulative sample distribution histogram w.r.t. positional $R$ error $\sigma_{\alpha, \delta}^{R-T}$. Large solid = total source population; smaller solid subpopulation $=$ within ROSAT error specification; dotted $=$ not within ROSAT error specification.

in Fig. 21. In order to account for the unmodelled errors, a basic error of $6^{\prime \prime}$ is routinely added. This explains the vanishing frequencies below $6^{\prime \prime}$ in Fig. 21. The mixture proportions are 0.55 and 0.45 but the distribution form is the same which is clear from the generation of the standard errors. In the light of the catalogue comparison made, the ROSAT position and positional errors passed the test; more than $50 \%$ of all sources lie not more than $20^{\prime \prime}$ away from the target position and are within the ROSAT error specification. The proportion of the latter compared to the remaining ones is still acceptable. The positional imprecisions found in simulations and in catalogue comparison differ by about $8^{\prime \prime}$. An offset of that size was expected. Our simulations were in pointed observations mode whereas ROSAT was in survey observation mode in data acquisition and in data processing. Here all the errors mentioned in Sect. 2 are involved and additional ones stemming from the

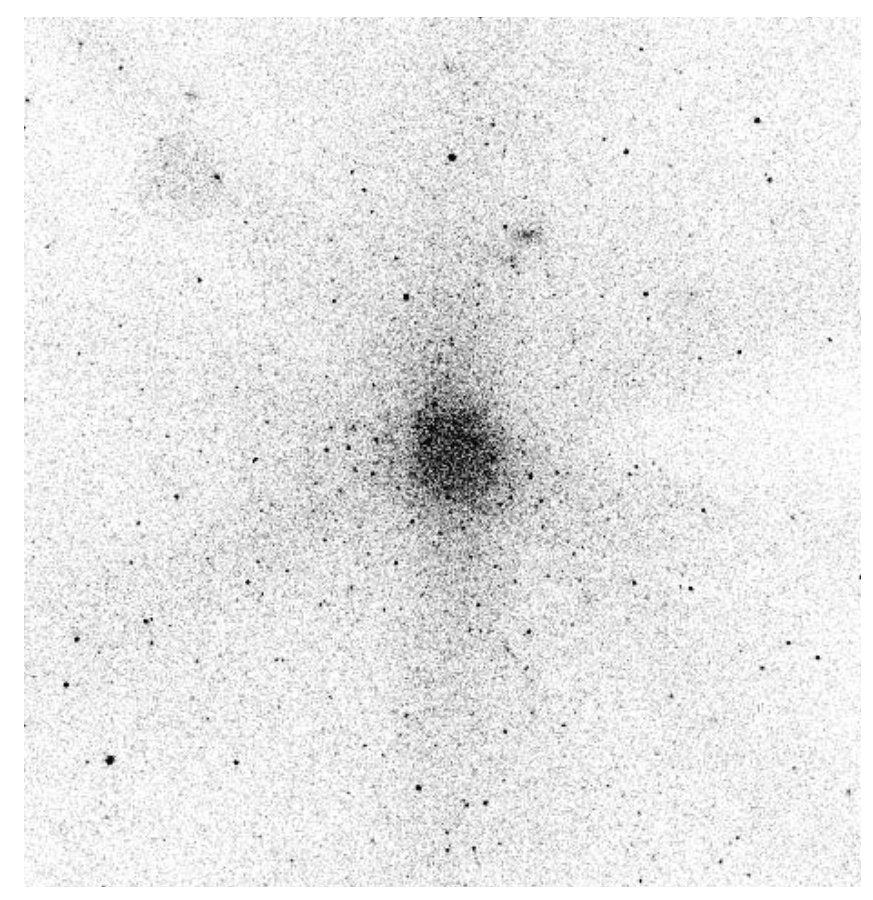

Fig. 22. A $6.1^{\circ} \times 6.1^{\circ}$ region around the ecliptic north pole observed in the ROSAT all-sky survey in the energy band $[0.1,2.35] \mathrm{keV}$. The central exposure time is $15.5 \mathrm{ks}$.

comparison. Taking account of all these factors, the mentioned additional imprecision is not considered harmful.

\section{Examples of ML source estimations}

In practice, MLE was tested many times. The ROSAT All-Sky Survey (RASS) described by Voges et al. $(1992,1999)$ is an instance. Almost 10000 pointed ROSAT observations were processed by MLE as described, see for example the group of contributions annotated by Trümper (1991).

We apply MLE to a sky field with many point sources. Figure 22 shows a $6.1^{\circ} \times 6.1^{\circ}$ field around the ecliptic north pole observed during the ROSAT All-Sky Survey in the energy band $[0.1,2.35] \mathrm{keV}$. The exposure time of the image centre was $15.5 \mathrm{ks}$. Only the photons which fell into the inner 19 arcmin of the ROSAT PSPC field of view were selected resulting in the image Fig. 22. The darker strips emanating from the central darker region are caused by a varying exposure time due to the ROSAT survey attitude. Figure 23 shows the same region augmented by point source positions. The small dark circles indicate the 545 point source positions found by MLE.

\section{Discussion}

The simulations in Sect. 4 were based on the source statistics in the ROSAT All-Sky Survey Bright Source Catalogue (BSC) and in the Faint Source Catalogue (FSC). Table 4 shows catalogue source mean values and a few ancillary quantities. From Table 4, the simulation parameters used can be considered typical for the ROSAT observations. The number $\Lambda_{S}=200$ of source photons taken in Sect. 4 for the sources with varying off-axis angle $\epsilon_{\mathrm{S}}$ was based on the 136 counts from Table 4 but 


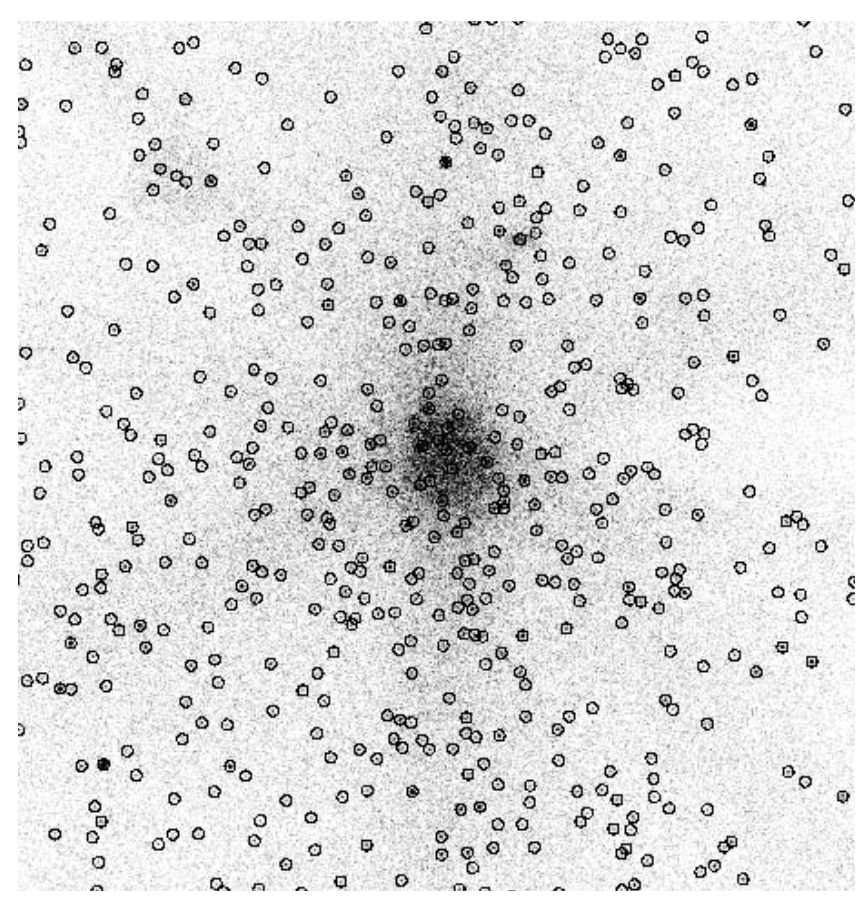

Fig. 23. The ecliptic north polar region as in Fig. 22. Circles indicate point source positions as found by MLE.

Table 4. The ROSAT All-Sky Survey catalogue mean source parameters. $\hat{\mu}_{\mathrm{BSC}}=$ Bright Source Catalogue means, $\hat{\mu}_{\mathrm{FSC}}=$ Faint Source Catalogue means.

\begin{tabular}{lrr}
\hline \hline Source parameter & $\hat{\mu}_{\text {BSC }}$ & $\hat{\mu}_{\text {FSC }}$ \\
\hline Source counts $/[\mathrm{cts}]$ & 135.63 & 25.38 \\
Source count rate $/\left[10^{-3} \mathrm{cts} \mathrm{s}^{-1}\right]$ & 311.70 & 12.35 \\
BKG count rate $/\left[10^{-3} \mathrm{cts} \mathrm{s}^{-1}\left({ }^{\prime}\right)^{-2}\right]$ & 1.19 & 1.02 \\
Source exposure time/[s] & 467.87 & 693.52 \\
Total source extension/["] & 23.00 & 21.10 \\
\hline
\end{tabular}

actually taken about $50 \%$ larger. The analogous precision simulations for ROSAT's survey observation mode are still lacking.

Not all observed ROSAT sources were point sources or circularly disk-shaped. A strategy for how to cope with sources of less simple morphology in an automated processing was devised by Voges et al. (1999). First, they are flaggged as exceptions indicating the kind of exception as precisely as possible. Afterwards, they are treated individually with more advanced software i.e. EXSAS. For groups of neighbouring point sources as discussed in Sect. 4.6, see the tools provided by EXSAS mentioned in Paper II.

Assume a perturbation not in the class $\Pi^{\prime}(\sigma)$ with the two perturbation parameters with up to $100 \sigma_{\mathrm{B}} \%$ in background and $100 \sigma_{P} \%$ in PSF with $\sigma_{\mathrm{B}} \neq \sigma_{P}$ allowed. Then the usage of lemma 2 with $\sigma_{\lambda}:=\max \left\{\sigma_{\mathrm{B}}, \sigma_{P}\right\}$ is the best possible choice. This gives an overestimation for $|\delta L / L|$ when both quantities are markedly unequal.

The ROSAT log-likelihood $L$ from Eq. (23) is given explicitly in terms of the design vector $q:=\left(\lambda_{\mathrm{B}}, p, V\right)$ and allows to include the perturbation with respect to the vignetting factor $V$, too.
In astronomy, the Bayesian approach is also applied to background estimation and source detection, cf. the contributions to the theme in Babu \& Feigelson $(1992,1999)$.

\section{Conclusions}

The notion "error", as used here, was explained and the errors were categorized. A group of error sources present in any X-ray observatory-data analyzer system was identified. The concept of a family of systems around a nominal system specified by a nominal design vector has been developed. Under source estimation via the Maximum Likelihood Principle to the family of systems, a family of log-likelihood functionals corresponds. A linear perturbation analysis with respect to a design vector comprising some or all of the error sources was set forth. The author is unaware of competing treatments of error propagation through the instrument. For the ROSAT observatory, a more detailed treatment of the influence of background and PSF perturbations on the source parameters and the log-likelihood value was carried out.

For the ROSAT observatory, the foregoing approaches were complemented with simulations. Through the analysis of simulated observations the precision figures were found, discussed and presented. The source count imprecision was found to be below 2 counts (for typical ROSAT source and background), positional imprecision below $2^{\prime \prime}$, and imprecision in source extent below $3^{\prime \prime}$ for both ROSAT detectors. For cross validation and in order to assess the over-all performance, the positions in the ROSAT All-Sky Bright Source Catalogue were measured from the optical TYCHO star catalogue counterparts. The positional imprecisions obtained in catalogue comparisons were by about $8^{\prime \prime}$ larger than those from simulations. This is in qualitative agreement with the foreseen 6 " additive positional error. Fortunately, the relative positioning of sources in the same observation is free of the attitude error.

Having tested the principles of ROSAT source estimation and their ROSAT implementation here (and in Papers I, II) with respect to their precision, we may conclude: the results confirm both principles and implementation.

Acknowledgements. The author would like to thank H.-U. Zimmermann for many constructive discussions and much valued comments, and for painstakingly reading parts of the manuscript. Figure 1 and Table 4 is a data courtesy of W. Voges. Figures 19-21 arose in a collaboration with him. The number of photons gathered with ROSAT's detectors in both ROSAT observation modes was extracted from the ROSAT data bases by J. Paul, supported by R. Gruber and W. Voges, all from the author's institute. In Paper II, the ROSAT launch was erroneously dated one month too late; June 1, 1990 is correct.

\section{References}

Babu, G. J., \& Feigelson, E. D. 1992, 1999, Statistical Challenges in Modern Astronomy I \& II (New York: Springer)

Boese F. G. 2000, A\&AS, 141, 507

Boese F. G., \& Döbereiner, S. 2001, A\&A, 370, 649 
Efron, B., \& Tibshirani, R. J. 1993, An Introduction to the Bootstrap (New York: Chapmann \& Hall)

Høg, E., Kuzmin, A., Bastian, U., et al. 1998, A\&A, 132, 155

Jaschek, C., Murtagh, F. 1990, Errors, Bias and Uncertainties in Astronomy (Cambridge: Cambridge University Press)

Lang, S. 1983, Real Analysis Addison-Wesley, Reading MA

O'Hagan, A. 1994, Kendall's Advanced Theory of Statistics: Bayesian Inference, Vol. 2B (London: Arnold)

Pfeffermann, E., Briel, U. G., \& Freyberg, M. J. 2003, Design and in-orbit-performance of the position sensitive proportional counter onboard the X-ray astronomy satellite ROSAT, Nuclear Instruments and Methods in Physics Research A, 515, 65

Trümper, J. 1983, Adv. Space Res. 241

Trümper, J. 1991, A\&A, 246, L1

Voges, W. 1992, The ROSAT all-sky X-ray Survey in Environment Observation and Climate Modelling through International Space Projects, Proceeding of the European International Space Year (ISY-3) Conference 1992, Munich, Germany, 30 March-4 April, Space in the service of the changing Earth, ed. T. Guyenne, \& J. J. Hunt, ESA Publication division, ESTEC, Nordwijk, The Netherlands, 3, 9
Voges, W. 1992, The ROSAT The Standard Analysis Software System in Environment Observation and Climate Modelling through International Space Projects, Proceeding of the European International Space Year (ISY-3) Conference 1992, Munich, Germany, 30 March-4 April, Space in the service of the changing Earth, ed. T. Guyenne, \& J. J. Hunt, ESA Publication division, ESTEC, Nordwijk, The Netherlands, 3, 223

Voges, W., Aschenbach, B., Boller, T., et al. 1999, 139, 389

Zimmermann, U., Boese, G., Becker, W., et al. 1998, EXSAS User's Guide: Extended Scientific Analysis System to Evaluate Data from the Astronomical X-ray Satellite ROSAT, Max-PlanckInstitut für extraterrestrische Physik, Garching bei München, Germany

Zimmermann, H. U., Belloni, T., et al. 1992, EXSAS: The interactive analysis system for ROSAT data in Environment Observation and Climate Modelling through International Space Projects, Proceeding of the European International Space Year (ISY-3) Conference 1992, Munich, Germany, 30 March-4 April, Space in the service of the changing Earth, ed. T. Guyenne, \& J. J. Hunt, ESA Publication division, ESTEC, Nordwijk, The Netherlands, 3, 231 